Review Article

\title{
The Quality of Methodological and Reporting in Network Meta-Analysis of Acupuncture and Moxibustion: A Cross-Sectional Survey
}

\author{
Ting Yuan $\mathbb{D}^{1},{ }^{1}$ Jun Xiong $\mathbb{D}^{2},{ }^{2}$ Xue Wang, ${ }^{1}$ Jun Yang, ${ }^{1}$ Yunfeng Jiang, ${ }^{2}$ Xiaohong Zhou, ${ }^{2}$ \\ Kai Liao, ${ }^{2}$ and Lingling $\mathrm{Xu}^{2}$ \\ ${ }^{1}$ Institute of Acupuncture, Moxibustion and Tuina, Jiangxi University of Traditional Chinese Medicine, Nanchang, Jiangxi, China \\ ${ }^{2}$ Department of Acupuncture and Moxibustion, The Affiliated Hospital of Jiangxi University of TCM, Nanchang, \\ Jiangxi, China
}

Correspondence should be addressed to Jun Xiong; xiongjun196071@163.com

Received 21 June 2020; Revised 26 September 2020; Accepted 26 December 2020; Published 11 January 2021

Academic Editor: Francesca Mancianti

Copyright (c) 2021 Ting Yuan et al. This is an open access article distributed under the Creative Commons Attribution License, which permits unrestricted use, distribution, and reproduction in any medium, provided the original work is properly cited.

Background. Acupuncture had long been a primary treatment in the healthcare system of China. In recent years, there were more and more network meta-analyses (NMAs) in the field of acupuncture and moxibustion, but the quality evaluation of NMAs was rare. Objectives. The goal of this study was to evaluate the methodological and reporting quality of NMAs and summarize the effects of different treatments of acupuncture and moxibustion. Methods. PubMed, Embase, the Cochrane Library, China National Knowledge Infrastructure Database (CNKI), WanFang Database (WF), Chinese Scientific Journal Database (VIP), and Chinese Biomedical Literature Database (CBM) were searched from inception to January 2020 without any language restriction. In addition, the unpublished studies and the references of initially included literature were also retrieved manually. We included all relevant NMAs treated with acupuncture and moxibustion; other therapies such as traditional Chinese medicine and Western medicine may also be included, but at least three types fall under the category of acupuncture in each NMA. Outcome indicators were not limited. We selected AMSTAR2 and PRISMA-NMA to evaluate the methodological and reporting quality of eligible studies, respectively. Results. In total, 29 NMAs were included finally, including 12 Chinese references and 17 English references. All eligible studies were published from May 2013 to August 2019 . The number of interventions was between 4 and 22. The number of clinical trials included ranged from 10 to 121 , with a total of 1098 clinical trials. The NMAs were involved in up to 23 diseases, knee osteoarthritis and primary dysmenorrhea covered with 3 NMAs separately, others focusing on chronic functional constipation, lumbar disc herniation, chronic fatigue syndrome, and the like. The Jadad scale and RoB scale were used as the bias risk assessment tools. Among them, 7 articles adopted the Jadad scale, 22 articles adopted the RoB scale (1 article adopted both the Jadad scale and RoB scale), and only 1 article did not mention the risk assessment tool. The AMSTAR2 methodological evaluation showed that the highest score was 13.5 points and the lowest was 4 , with an average of 8.64 and a median of 9.5. According to the quality criteria, only one of them was in high quality, twenty-four were in medium quality, and four were in low quality. The PRISMA-NMA reporting quality evaluation showed that the highest score was 29 points and the lowest was 13.5, with an average of 23.62 and a median of 24.5; severe flaws also existed in some items, especially in "Structured summary," "Protocol and registration," "Search," "Data collection process," "Data items," "Additional analyses," "Risk of bias across studies," and "Results of additional analyses." Conclusion. The number of NMAs in the field of acupuncture and moxibustion was still in the initial stage. Overall, their methodology and reports were of moderate quality. However, severe flaws also existed in some items. Because the eligible NMAs were limited, the conclusion needed further research to confirm its authenticity and reliability. 


\section{Introduction}

Acupuncture has a history of more than 4,000 years and is now commonly used in medical care in China. As a traditional oriental therapy, it has been widely used globally [1]. With the development of evidence-based acupuncture and moxibustion, acupuncture and moxibustion is more and more popular in clinics because of its simple treatment, quick effect, and nontoxic side effects. However, there are many kinds of acupuncture and moxibustion intervention methods, and the indications are similar. Traditional metaanalysis can only achieve pairwise direct comparison of intervention measures, but it cannot carry out indirect comparison of intervention measures without a direct comparative study, and let alone comparative analysis of various intervention measures; so, it is difficult to solve this problem.

Network meta-analyses (NMAs) are still named multiple-treatments meta-analysis (MTM) or mixed treatment comparison meta-analyses (MTC). Meta-analysis is an analytical method that evaluates the relative efficiency of treatments and synthesizes evidence using a randomized controlled trial network diagram. This method is based on the combination of traditional direct/head-to-head comparison and indirect comparison for meta-analysis, which can simultaneously compare the efficacy of three or more interventions. It was developed on the basis of classical metaanalysis, which resulted in a consistent and complete set of comparisons based on all available evidence from existing research studies [2-5]. The NMA provides evidence for clinical drug selection by quantifying different interventions to treat the same disease and ranking the benefits [6]. As an emerging evidence synthesis tool, NMAs are becoming more and more popular, which can make more decisions or choices than classic meta-analysis [7-10].

In recent years, NMA has made some progress in the field of acupuncture and moxibustion, and the number of publication of NMAs related to acupuncture and moxibustion is on the rise, but its quantity is still small, and its quality still lacks systematic evaluation. This study through retrieving NMAs of acupuncture and moxibustion published a comprehensive understanding of the present situation, and apply AMSTAR2 (a measurement tool to assess the methodological quality of systematic reviews) and PRISMA-NMA (PRISMA extension statement for reporting of systematic reviews incorporating network meta-analyses of health care interventions: checklist and explanations) to assess the methodological and reporting quality of the published NMAs in acupuncture and moxibustion field systematically, in order to offer reference to improve the quality of acupuncture and moxibustion in NMA.

\section{Materials and Methods}

2.1. Information Sources. PubMed, Embase, the Cochrane Library, China National Knowledge Infrastructure Database (CNKI), WanFang Database (WF), Chinese Scientific Journal Database (VIP), and Chinese Biomedical Literature Database (CBM) were searched from inception to January
2020 without any language restriction. In addition, the unpublished studies and the references of initially included literature were also retrieved manually. The comprehensive search strategy for PubMed is presented in Table 1. The retrieval of other electronic databases was similar to PubMed, which adopts the combination of subject words and keywords.

2.2. Eligible Criteria. We included all relevant NMAs treated with acupuncture and moxibustion; other therapies such as traditional Chinese medicine and Western medicine may also be included, but at least three types fall under the category of acupuncture in each NMA. Outcome indicators were not limited, while language limited in Chinese and English.

2.3. Exclusion Criteria. (1) Duplicate detection and republished literature. (2) Theoretical research. (3) Reviews, conference papers, and abstracts. (4) Incomplete data of the results. (5) Acupuncture interventions <3. (6) Non-Chinese and English literature.

2.4. Study Selection and Data Extraction. The search was conducted by NoteExpress 5.3.0 literature management software. NoteExpress 5.3.0 automatic duplicate check function was used and combined with manual duplicate check to eliminate the duplicate research. By reading the title and abstract, we excluded studies that obviously do not meet the inclusion criteria; downloaded and read the full text of the remaining studies to judge; and for research with incomplete data report, tried to contact the author and gain the complete data. The three evaluators (TY, XW, and JY) screened and extracted the literatures back to back independently according to the inclusion and exclusion criteria, and cross-checked the results. The included details were as follows: author information, year of publication, sample size, disease, type of study, diagnostic criteria, number of original study, description of interventions, number of interventions, comparators, outcome measures, and risk assessment tools for bias and adverse reactions. When there was any disagreement, it was resolved by the fourth researcher (JX).

2.5. Methodological and Reporting Quality Assessment Tools. Three independent researchers (TY, XW, and JY) evaluated the methodological and reporting quality back to back. The AMSTAR2 tool contained 16 aspects [11]. For each aspect, when the answer was "yes," the score was 1 , and when the answer was "no," "cannot answer," or "not applicable," the score was 0 . The total score of an NMA was calculated by counting the number of "yes" in 16 items on a scale of $0-16$. A score of 12-16 was rated as "high quality," a score of 7-11 was rated as "medium quality," and a score of 0-6 was rated as "low quality." The PRISMA-NMA contained 32 items [12]. For each item, a score of " 1 " means full compliance, " 0.5 " means partial compliance, and " 0 " means noncompliance $[13,14]$. The total PRISMA-NMA score of an NMA was calculated by accumulating the scores of each item, with 
TABLE 1: Search strategy.

\begin{tabular}{|c|c|}
\hline Source: & PubMed; searched on: January 22, 2020 \\
\hline Search & Query \\
\hline \#1 & "acupuncture" [Ti/Ab] OR “moxibustion” [Ti/Ab] \\
\hline \#2 & $\begin{array}{c}\text { "network meta-analysis" }[\mathrm{Ti} / \mathrm{Ab}] \text { OR "mixed treatment meta-analysis" [Ti/Ab] OR "multiple treatment comparison meta-analysis" } \\
{[\mathrm{Ti} / \mathrm{Ab}] \text { OR "bayes meta-analysis" [Ti/Ab] OR "indirect comparison" [Ti/Ab] }}\end{array}$ \\
\hline \#3 & "network meta-analysis" [MeSH] \\
\hline \#4 & "acupuncture" [MeSH] OR "moxibustion" [MeSH] \\
\hline \#5 & $\# 1$ AND \#2 \\
\hline \#6 & \#3 AND \#4 \\
\hline \#7 & \#5 OR \#6 \\
\hline
\end{tabular}

a range of 0-32. A score of 26-32 was rated as "high quality," a score of 20-25.5 as rated as "medium quality," and a score of $0-19.5$ as rated as "low quality." When there was any disagreement, it was resolved by the fourth researcher (JX).

2.6. Data Analysis. We analyzed the characteristics of included studies through descriptive statistical methods. All the data used were counted in the Excel 2007 spreadsheet. We described the dichotomous data in terms of number and percentage and the continuous variables in terms of median with interquartile range (IQR). And we calculated the number of papers per item, its percentage, and $95 \%$ confidence intervals. We summarized the scores according to the quality evaluation tool. AMSTAR2 and PRISMA-NMA scored 16 and 32, respectively. Finally, we calculated the total score through adding a list of each component.

\section{Results}

3.1. Search Results. 242 related references through searching seven electronic databases and manual searches were retrieved. After reading the title and abstract, a total of 29 studies were included [15-43]. The literature screening process is shown in Figure 1.

3.2. Study Characteristics. The characteristics of all included NMAs were documented. After screening, 29 NMAs finally met the inclusion criteria, including 12 Chinese references (including 1 master's thesis) and 17 English references. All eligible studies were published from May 2013 to August 2019. The study contained 4-22 types of treatments and 10-121 RCTs for 1098 RCTs in total. The NMAs were involved in up to 23 diseases, knee osteoarthritis and primary dysmenorrhea covered with 3 NMAs separately, others focusing on chronic functional constipation, lumbar disc herniation, chronic fatigue syndrome, and the like. The Jadad scale and RoB scale were used as the bias risk assessment tools. Among them, 7 articles adopted the Jadad scale, 22 articles adopted the RoB scale (1 article adopted both the Jadad scale and RoB scale), and only 1 article did not mention the risk assessment tool. The characteristics of the eligible studies are presented in Table 2.

3.3. Methodological Quality Assessment. According to the AMSTAR2 checklist, the median score and IQR of eligible
NMAs was 9.5 (6.5-10.75), and the details are presented in Tables 3 and 4 . The item with the best degree of compliance was "comprehensive literature search" (100\%) (Table 4). Longitudinal analysis, a good degree of compliance was with item 9 (93.1\%), item $11(79.31 \%)$, and items 1, 5, and 6 (75.86\%). However, a poor degree of compliance was with item $2(17.24 \%)$ and item $3(6.9 \%)$, and the worst degree of compliance was with item $7(0 \%)$ and item $10(0 \%)$. The details are presented in Tables 3 and 4 and Figure 2.

3.4. Reporting Quality Assessment. According to the PRISMA-NMA checklist, the median score and IQR of eligible NMAs was $24.5(20.5-26.5)$. The item with the best degree of compliance was item 1 "Title" (100\%). Longitudinal analysis, a good degree of compliance was with item 4 (96.55\%), item $12(96.55 \%)$, item 25 (96.55\%), item 18 (93.10\%), item 26 (93.10\%), item 3 (89.65\%), item 19 (89.65\%), and item 24 (86.21\%). However, a poor degree of compliance was with item 23 (27.59\%), item 2 (17.24\%), item 5 (17.24\%), item 10 (17.24\%), and item 11 (10.34\%), and the worst degree of compliance was with item S4 (3.45\%). There are significant problems in the reporting methods and results sections, such as underreporting or selective reporting. Three studies $[29,39,42](10.34 \%)$ reported evaluating the risk of bias within individual studies in the methods section (item 12), but did not really evaluate it in the results section (item 19). Horizontal analysis, there were four NMAs (13.79\%) that scored less than 20 points, with a minimum score of 13.5. The details are presented in Tables 5 and 6 .

\section{Discussion}

4.1. Summary of Main Findings. The goal of this crosssectional survey was to evaluate the methodological and reporting quality of NMAs and summarize the effects of different treatments of acupuncture and moxibustion (Table 6). A total of 29 acupuncture NMAs were included in this study. From the perspective of the number and publication time, the development of NMA in acupuncture and moxibustion was still in the initial stage, with a small number, but it had shown a trend of gradual growth. In terms of disease, more than half were chronic pain. In terms of intervention measures, more than half were acupuncture combined therapy. It was not difficult to find that NMAs of acupuncture and moxibustion was still limited to several diseases, and there were still large gaps in many aspects. 


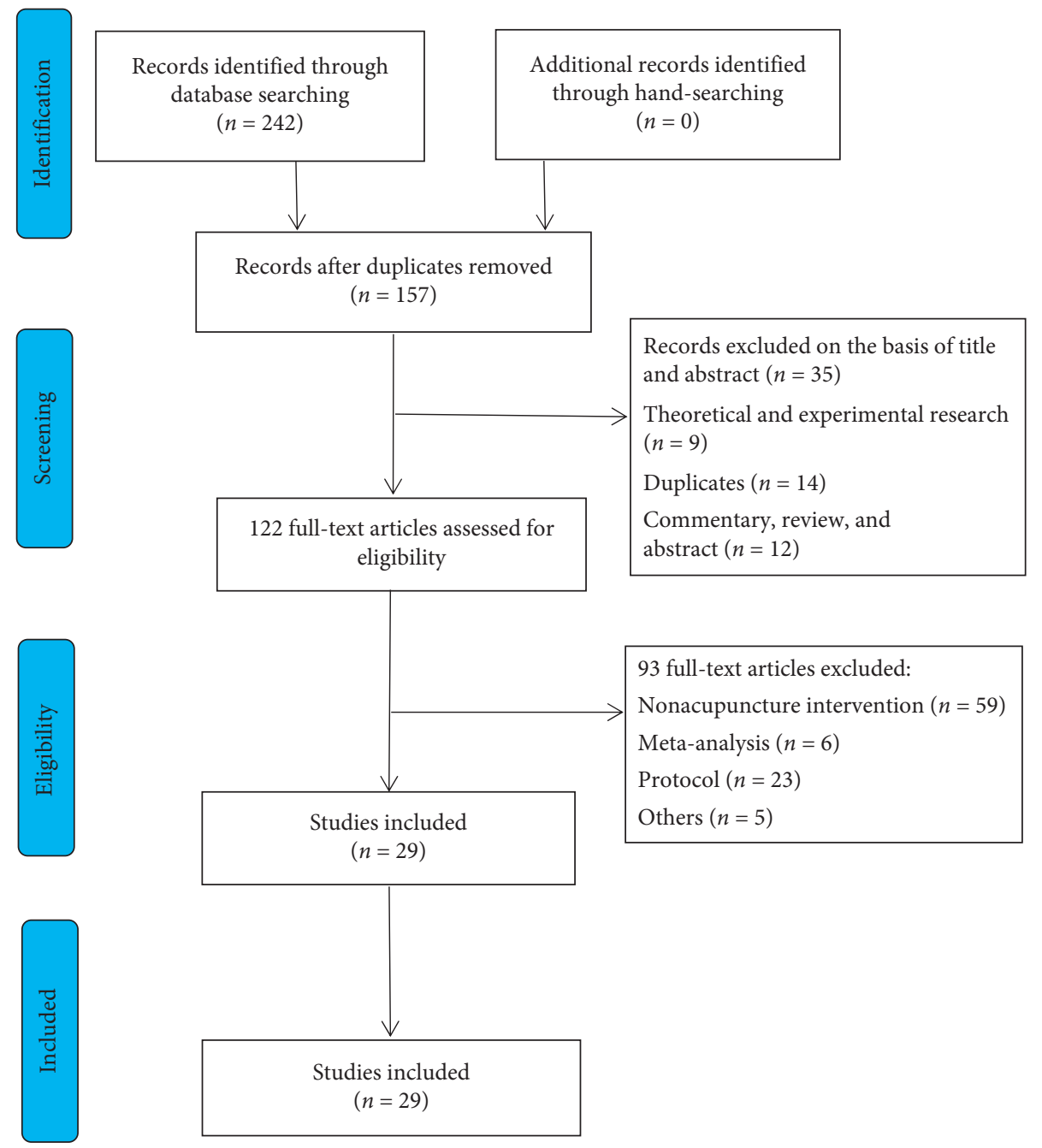

FIGURE 1: Flow diagram of the study.

The methodological quality of NMAs was important, so we evaluated the methodological quality of NMAs in acupuncture and moxibustion according to AMSTAR2 tool. The results showed some methodological deficiencies, particularly with regard to item 2 , item 3 , item 7 , and item 10 . The highest NMA score for each item was 13.5, the lowest was 2.5, and the median and IQR was 9.5 (6.5-10.75), indicating average methodological quality.

The quality evaluation of the report showed that the quality of acupuncture NMAs was generally acceptable, indicating that the NMA researchers had a high level of evidence-based medical knowledge and scientific research literacy. Some items need to be improved, particularly with regard to the structured summary (item 2), protocol and registration (item 5), search (item 8), data collection process (item 10), data items (item 11), additional analyses (item 16), risk of bias across studies (Results section) (item 22), and results of additional analyses (item 23 ). From the perspective of a single NMA, the highest score was 29, the lowest was only 13.5 , and the median and IQR was 9.5 (6.5-10.75), showing that the quality of reports included in the study was of average quality. PRISMA-NMA checklist was helpful to improve the reporting quality of acupuncture NMAs. Therefore, it was necessary to improve the comprehensiveness and standardization of the report.

4.2. Strengths and Limitations. First, this was the first study that evaluated the methodological and reporting quality of NMAs comprehensively, which complied with the methodological and reporting guidelines in the field of acupuncture and moxibustion. Even though there were two studies regarding the methodological or reporting quality of NMAs in TCM, one English article excluded acupuncture and moxibustion [44] and one Chinese article included acupuncture and moxibustion literature incompletely [45]. Second, compared with published quality studies of NMAs in acupuncture and moxibustion, this review implemented a more comprehensive and detailed literature retrieval strategy. In addition, the unpublished studies and the references initially were also retrieved manually. As a result, the results were more credible. 
TABLE 2: Basic characteristics of eligible studies.

\begin{tabular}{|c|c|c|c|c|c|c|c|}
\hline $\begin{array}{l}\text { Study } \\
\text { ID }\end{array}$ & Disease & $\begin{array}{l}\text { Sample } \\
\text { size }\end{array}$ & $\begin{array}{l}\text { Number of } \\
\text { interventions }\end{array}$ & Description of interventions & $\begin{array}{l}\text { Number } \\
\text { of RCT }\end{array}$ & Outcome & $\begin{array}{l}\text { Risk } \\
\text { assessment } \\
\text { tool }\end{array}$ \\
\hline $\begin{array}{l}\text { Fu et al. } \\
{[15]}\end{array}$ & $\begin{array}{l}\text { Acute gouty } \\
\text { arthritis }\end{array}$ & 4931 & 10 & $\begin{array}{c}\text { Western medicine, } \\
\text { acupuncture + pricking blood and } \\
\text { cupping, Chinese medicine + cupping, } \\
\text { acupuncture and moxibustion, Chinese } \\
\text { medicine, Western medicine + pricking } \\
\text { blood and cupping, pricking blood and } \\
\text { cupping, Western } \\
\text { medicine + acupuncture, Western } \\
\text { medicine + massage, and Western } \\
\text { medicine + Chinese medicine }\end{array}$ & 66 & Effective rate & Jadad \\
\hline $\begin{array}{l}\text { Ding } \\
{[16]}\end{array}$ & $\begin{array}{l}\text { Abdominal } \\
\text { distension } \\
\text { after } \\
\text { abdominal } \\
\text { operation }\end{array}$ & 2047 & 7 & $\begin{array}{c}\text { Acupoint application, acupoint injection, } \\
\text { acupoint massage, moxibustion, TCM } \\
\text { enema, TCM hot ironing, and routine } \\
\text { nursing }\end{array}$ & 22 & Effective rate & Jadad \\
\hline $\operatorname{Li}[17]$ & $\begin{array}{l}\text { Poststroke } \\
\text { depression }\end{array}$ & 1895 & 13 & $\begin{array}{c}\text { Acupuncture + Chinese medicine, ear } \\
\text { bean + Chinese medicine, electric } \\
\text { acupuncture + Chinese medicine, } \\
\text { moxibustion + Chinese medicine, } \\
\text { auricular acupoint } \\
\text { electroacupuncture + Chinese medicine, } \\
\text { head acupuncture + acupoint embedding, } \\
\text { auricular acupoint } \\
\text { electroacupuncture + acupuncture, ear } \\
\text { bean + acupuncture, } \\
\text { acupuncture + acupoint injection, } \\
\text { acupuncture + wuxing music, wuxing } \\
\text { music + Chinese medicine, SSRIs, and } \\
\text { acupuncture + moxibustion } \\
\end{array}$ & 23 & Effective rate & Jadad \\
\hline $\begin{array}{l}\text { Bu et al. } \\
{[18]}\end{array}$ & Optic atrophy & 1369 & 11 & $\begin{array}{c}\text { Western medicine, acupuncture, } \\
\text { acupuncture + moxibustion, ear } \\
\text { point + Chinese medicine, } \\
\text { electroacupuncture, } \\
\text { acupuncture + Chinese and Western } \\
\text { medicine, moxibustion + Chinese and } \\
\text { Western medicine, } \\
\text { electroacupuncture + Chinese and } \\
\text { Western medicine, Chinese and Western } \\
\text { medicine, Chinese medicine, and } \\
\text { acupuncture + Western medicine }\end{array}$ & 16 & $\begin{array}{l}\text { Effective rate, } \\
\text { vision, horizon, } \\
\text { and visual } \\
\text { evoked potential }\end{array}$ & RoB \\
\hline $\begin{array}{l}\text { Zhang } \\
{[19]}\end{array}$ & $\begin{array}{l}\text { Ankylosing } \\
\text { spondylitis }\end{array}$ & 2208 & 9 & $\begin{array}{l}\text { Sulfasalazine, acupuncture, moxibustion, } \\
\text { bee acupuncture, } \\
\text { acupuncture }+ \text { moxibustion, } \\
\text { acupuncture }+ \text { moxibustion }+ \text { cupping, } \\
\text { and moxibustion + bee needle }\end{array}$ & 25 & Effective rate & RoB \\
\hline $\begin{array}{l}\text { Song } \\
{[20]}\end{array}$ & $\begin{array}{l}\text { Polycystic } \\
\text { ovary } \\
\text { syndrome }\end{array}$ & 4605 & 14 & $\begin{array}{l}\text { Acupuncture-medication therapy, } \\
\text { Western medicine, acupuncture and } \\
\text { moxibustion, acupuncture, } \\
\text { acupuncture + ear points, } \\
\text { moxibustion + Chinese medicine, } \\
\text { acupuncture + ear points, } \\
\text { acupuncture + placebo, } \\
\text { placebo + Western medicine, Chinese } \\
\text { medicine + Western medicine, placebo, } \\
\text { acupoint thread-embedding therapy and } \\
\text { medication, Chinese medicine, and } \\
\text { moxibustion }\end{array}$ & 39 & $\begin{array}{l}\text { Ovulation rate } \\
\text { and pregnancy } \\
\text { rate }\end{array}$ & RoB \\
\hline
\end{tabular}


TABle 2: Continued.

\begin{tabular}{|c|c|c|c|c|c|c|c|}
\hline $\begin{array}{l}\text { Study } \\
\text { ID }\end{array}$ & Disease & $\begin{array}{l}\text { Sample } \\
\text { size }\end{array}$ & $\begin{array}{l}\text { Number of } \\
\text { interventions }\end{array}$ & Description of interventions & $\begin{array}{l}\text { Number } \\
\text { of RCT }\end{array}$ & Outcome & $\begin{array}{l}\text { Risk } \\
\text { assessment } \\
\text { tool }\end{array}$ \\
\hline $\begin{array}{l}\text { Yang } \\
\text { et al. } \\
{[21]}\end{array}$ & $\begin{array}{l}\text { Lumbar disc } \\
\text { herniation }\end{array}$ & 2589 & 4 & $\begin{array}{l}\text { Acupuncture, acupuncture + cupping, } \\
\text { acupuncture + massage, and } \\
\text { acupuncture }+ \text { cupping + massage }\end{array}$ & 30 & $\begin{array}{c}\text { Pain } \\
\text { improvement, } \\
\text { effectiveness, } \\
\text { cure rate, } \\
\text { recurrence rate, } \\
\text { and economic } \\
\text { indicators. }\end{array}$ & RoB \\
\hline $\operatorname{Li}[22]$ & $\begin{array}{c}\text { Primary } \\
\text { dysmenorrhea }\end{array}$ & 4600 & 6 & $\begin{array}{c}\text { Acupuncture, acupuncture }+ \\
\text { moxibustion, acupuncture }+ \text { indirect } \\
\text { moxibustion, warm acupuncture, } \\
\text { electroacupuncture, and } \\
\text { electroacupuncture + warm acupuncture }\end{array}$ & 56 & $\begin{array}{l}\text { Effective rate, } \\
\text { VAS, and } \\
\text { dysmenorrhea } \\
\text { symptom score }\end{array}$ & RoB \\
\hline
\end{tabular}

Acupoint catgut embedding, acupoint catgut embedding + loratadine, acupoint Jia et al. Chronic $\quad 1186 \quad 9 \quad$ catgut embedding + mizolastine, acupoint [23] urticaria $1186 \quad 9 \quad$ catgut embedding + cetirizine, acupoint catgut embedding + epstein, loratadine, mizolastine, cetirizine, and epstein

Hyaluronic acid (HA), needle knife, Liu [24] $\begin{gathered}\text { Knee } \\ \text { osteoarthritis }\end{gathered} 3417 \quad 6 \quad \begin{gathered}\text { myaluronic acid (HA), needle knife }+ \text { HA, needle knife + interna } \\ \text { medicine, needle knife + massage, and } \\ \text { needle knife + acupuncture }\end{gathered}$ needle knife + acupuncture medicine therapy, multipoint + Western $\begin{array}{lccc}\text { Feng } & \text { Primary } & 10259 & 9\end{array}$ multipoint, Western medicine routine
therapy, and placebo medicine conventional therapy, single point + TCM, TCM, single point,

Rehabilitation, acupuncture, acupuncture + rehabilitation, acupuncture + massage, acupuncture + TCM

Liu [26] Poststroke $1898 \quad 9 \quad$ fumigation + rehabilitation,
acupuncture + massage + cupping, acupuncture + massage + rehabilitation, acupuncture $+\mathrm{TCM}+$ rehabilitation, and acupuncture + joint loosening + rehabilitation

HRT, acupuncture, moxibustion, needlewarming moxibustion, electroacupuncture, catgut implantation at acupoint , TCM, acupuncture + TCM, moxibustion + TCM,

\begin{tabular}{|c|c|c|}
\hline $\begin{array}{l}\text { Yang } \\
\text { et al. } \\
{[27]}\end{array}$ & $\begin{array}{c}\text { Premature } \\
\text { ovarian } \\
\text { insufficiency }\end{array}$ & 3046 \\
\hline
\end{tabular}
electroacupuncture + TCM, acupoint application + TCM, auricular point sticking + TCM, acupressure + TCM, catgut implantation at acupoint + TCM, acupuncture + HRT,

electroacupuncture + HRT, catgut implantation at acupoint $+\mathrm{HRT}$, and acupuncture + TCM + HRT

\begin{tabular}{lccccc}
\hline $\begin{array}{l}\text { Zhu } \\
\text { et al. }\end{array}$ & $\begin{array}{c}\text { Chronic } \\
\text { constipation }\end{array}$ & 11032 & \multirow{2}{*}{10} & $\begin{array}{c}\text { Acupuncture, polyethylene glycol, } \\
\text { lactulose, linaclotide, lubiprostone, } \\
\text { bisacodyl, prucalopride, sham } \\
\text { acupuncture, tegaserod, and placebo }\end{array}$ & $\begin{array}{c}\text { Symptoms of } \\
\text { chronic } \\
\text { constipation and } \\
\text { side effects }\end{array}$ \\
\hline
\end{tabular}


TABle 2: Continued.

\begin{tabular}{|c|c|c|c|c|c|c|c|}
\hline $\begin{array}{l}\text { Study } \\
\text { ID }\end{array}$ & Disease & $\begin{array}{l}\text { Sample } \\
\text { size }\end{array}$ & $\begin{array}{l}\text { Number of } \\
\text { interventions }\end{array}$ & Description of interventions & $\begin{array}{l}\text { Number } \\
\text { of RCT }\end{array}$ & Outcome & $\begin{array}{l}\text { Risk } \\
\text { assessment } \\
\text { tool }\end{array}$ \\
\hline $\begin{array}{l}\text { Zheng } \\
\text { [29] }\end{array}$ & $\begin{array}{l}\text { Chronic } \\
\text { functional } \\
\text { constipation }\end{array}$ & 4324 & 8 & $\begin{array}{l}\text { Acupuncture, mosapride, insoluble fiber, } \\
\text { massage, mineral water, probiotic, TENS, } \\
\text { and moxibustion }\end{array}$ & 33 & $\begin{array}{l}\text { Weekly stool } \\
\text { frequency, } \\
\text { Bristol score, } \\
\text { responder rate, } \\
\text { and adverse } \\
\text { event } \\
\end{array}$ & RoB \\
\hline Qin [30] & $\begin{array}{l}\text { Chronic } \\
\text { prostatitis/ } \\
\text { chronic pelvic } \\
\text { pain syndrome }\end{array}$ & 1203 & 7 & $\begin{array}{l}\text { Acupuncture, electroacupuncture, alpha- } \\
\text { blockers, antibiotics, dual therapy, sham } \\
\text { acupuncture, and placebo }\end{array}$ & 12 & $\begin{array}{l}\text { NIH-CPSI QoL } \\
\text { score }\end{array}$ & RoB \\
\hline $\begin{array}{l}\text { Li et al. } \\
\text { [31] }\end{array}$ & $\begin{array}{c}\text { Knee } \\
\text { osteoarthritis }\end{array}$ & 2065 & 7 & $\begin{array}{l}\text { Common manual acupuncture, } \\
\text { electroacupuncture, fire needle, warm } \\
\text { needle, placebo, sham needle, and } \\
\text { education }\end{array}$ & 16 & $\begin{array}{l}\text { WOMAC, } \\
\text { stiffness, and } \\
\text { physical } \\
\text { function scores }\end{array}$ & RoB \\
\hline $\begin{array}{l}\text { Mo et al. } \\
{[32]}\end{array}$ & $\begin{array}{l}\text { Lumbar disc } \\
\text { herniation }\end{array}$ & 13075 & 4 & $\begin{array}{l}\text { Tuina, traction, acupuncture, and Chinese } \\
\text { herbs }\end{array}$ & 121 & $\begin{array}{c}\text { Invalid rate, cure } \\
\text { rate, } \mathrm{VAS} \text {, and } \\
\text { JOA }\end{array}$ & RoB \\
\hline $\begin{array}{l}\text { Luo } \\
\text { et al. } \\
{[33]}\end{array}$ & $\begin{array}{c}\text { Primary } \\
\text { dysmenorrhea }\end{array}$ & 1511 & 8 & $\begin{array}{l}\text { Traditional acupuncture, eye } \\
\text { acupuncture, wrist-ankle acupuncture, } \\
\text { superficial acupuncture, moxibustion, } \\
\text { electroacupuncture, ear acupuncture, and } \\
\text { abdominal acupuncture }\end{array}$ & 17 & Effective rate & RoB \\
\hline $\begin{array}{l}\text { Yeh } \\
\text { et al. } \\
{[34]}\end{array}$ & Psoriasis & 869 & 6 & $\begin{array}{c}\text { Acupuncture, acupressure, acupoint } \\
\text { bloodletting, acupoint catgut embedding, } \\
\text { Chinese herbal medicine, and narrow- } \\
\text { band ultraviolet B }\end{array}$ & 10 & PASI and TCM & Jadad RoB \\
\hline $\begin{array}{l}\text { Chen } \\
\text { et al. } \\
\text { [35] }\end{array}$ & Migraine & 3656 & 9 & $\begin{array}{c}\text { Acupuncture, flunarizine, metoprolol, } \\
\text { propranolol, propranolol + flunarizine, } \\
\text { sham acupuncture, topiramate, usual care, } \\
\text { and waiting list }\end{array}$ & 19 & $\begin{array}{l}\text { Migraine } \\
\text { episodes, the } \\
\text { number of } \\
\text { migraine days, } \\
\text { migraine } \\
\text { frequency, } \\
\text { responder rate, } \\
\text { and adverse } \\
\text { event rate }\end{array}$ & RoB \\
\hline $\begin{array}{l}\text { Tan } \\
\text { et al. } \\
{[36]}\end{array}$ & $\begin{array}{c}\text { Essential } \\
\text { hypertension }\end{array}$ & 2649 & 15 & $\begin{array}{l}\text { Electroacupuncture, moxibustion, warm } \\
\text { needle therapy, sham acupuncture, } \\
\text { behavioral therapy, angiotensin- } \\
\text { converting enzyme inhibitors (ACEIs), } \\
\text { angiotensin receptor blockers (ARBs), } \\
\text { calcium channel blocker (CCB), beta- } \\
\text { blocker, acupuncture-combined ACEI, } \\
\text { acupuncture-combined CCB, } \\
\text { acupuncture-combined behavior, } \\
\text { electroacupuncture-combined CCB, sham } \\
\text { acupuncture, and nontreatment }\end{array}$ & 31 & $\begin{array}{l}\text { posttreatment } \\
\text { BP changes, } \\
\text { response rate, } \\
\text { and MACE }\end{array}$ & RoB \\
\hline $\begin{array}{l}\text { Li et al. } \\
\text { [37] }\end{array}$ & $\begin{array}{c}\text { Myofascial } \\
\text { pain syndrome }\end{array}$ & 1692 & 22 & $\begin{array}{l}\text { Placebo-sham, MA, EA, DN, MET, TCT, } \\
\text { MT, LTrP-I, MDIMST, MSN, TTM, BTX- } \\
\text { A-TrP-I, FN, SWAM, EA\&ESNC, SPM, } \\
\text { DN\&MET, BTrP-I, TrP-DN\&EDU, } \\
\text { Stretch, DN\&Stretch, laser, and PT }\end{array}$ & 33 & $\begin{array}{c}\text { Pain } \\
\text { measurement } \\
\text { (VAS, NRS, and } \\
\text { PPT), adverse } \\
\text { events (ROM), } \\
\text { and functional } \\
\text { status }\end{array}$ & RoB \\
\hline
\end{tabular}


TABLE 2: Continued.

\begin{tabular}{|c|c|c|c|c|c|c|c|}
\hline $\begin{array}{l}\text { Study } \\
\text { ID }\end{array}$ & Disease & $\begin{array}{l}\text { Sample } \\
\text { size }\end{array}$ & $\begin{array}{l}\text { Number of } \\
\text { interventions }\end{array}$ & Description of interventions & $\begin{array}{l}\text { Number } \\
\text { of RCT }\end{array}$ & Outcome & $\begin{array}{l}\text { Risk } \\
\text { assessment } \\
\text { tool }\end{array}$ \\
\hline $\begin{array}{l}\text { Zhu } \\
\text { et al. } \\
{[38]}\end{array}$ & $\begin{array}{l}\text { Diarrhea- } \\
\text { predominant } \\
\text { irritable bowel } \\
\text { syndrome }\end{array}$ & 9369 & 7 & $\begin{array}{l}\text { Acupuncture, sham acupuncture, } \\
\text { pinaverium bromide, alosetron, } \\
\text { eluxadoline, ramosetron, and rifaximin }\end{array}$ & 29 & $\begin{array}{l}\text { Effective rate, } \\
\text { side effects } \\
\text { (constipation } \\
\text { and rash), and } \\
\text { common } \\
\text { acupuncture } \\
\text { points. }\end{array}$ & Jadad \\
\hline $\begin{array}{l}\text { Xiong } \\
\text { and } \\
\text { Chen } \\
{[39]}\end{array}$ & $\begin{array}{l}\text { Diabetic } \\
\text { peripheral } \\
\text { neuropathy }\end{array}$ & 2602 & 7 & $\begin{array}{l}\text { Manual acupuncture, electroacupuncture, } \\
\text { needle knocking acupuncture, warm } \\
\text { needling and moxibustion, mecobalamin, } \\
\text { no interventions, and vitamin B }\end{array}$ & 40 & $\begin{array}{l}\text { global symptom } \\
\text { improvement }\end{array}$ & non \\
\hline $\begin{array}{l}\text { Yang } \\
\text { et al. } \\
{[40]}\end{array}$ & Heart failure & 2116 & 5 & $\begin{array}{l}\text { Acupuncture, moxibustion, acupoint } \\
\text { application, acupoint injection, and } \\
\text { warming acupuncture-moxibustion }\end{array}$ & 26 & HFC and LVEF & RoB \\
\hline $\begin{array}{l}\text { Zhang } \\
\text { et al. } \\
{[41]}\end{array}$ & Obesity & 2283 & 6 & $\begin{array}{l}\text { AAS, EA, ACE, WA, AR: acupuncture and } \\
\text { related therapies and combination of } \\
\text { acupuncture and related therapies. }\end{array}$ & 34 & $\begin{array}{l}\text { BW, BMI, and } \\
\text { adverse events }\end{array}$ & RoB \\
\hline $\begin{array}{l}\text { Corbett } \\
2013 \\
{[42]}\end{array}$ & $\begin{array}{c}\text { Knee } \\
\text { osteoarthritis }\end{array}$ & 9709 & 8 & $\begin{array}{l}\text { Interferential therapy, acupuncture, } \\
\text { TENS, pulsed electrical stimulation, } \\
\text { balneotherapy, aerobic exercise, sham } \\
\text { acupuncture, and muscle-strengthening } \\
\text { exercise }\end{array}$ & 114 & WOMAC pain & RoB \\
\hline $\begin{array}{l}\text { Wang } \\
\text { et al. } \\
{[43]}\end{array}$ & $\begin{array}{l}\text { Chronic } \\
\text { fatigue } \\
\text { syndrome }\end{array}$ & 2255 & 5 & $\begin{array}{l}\text { CbAM, SAM, Chinese herbal medicine, } \\
\text { Western medicine, and sham acupuncture }\end{array}$ & 31 & Effective rate & RoB \\
\hline
\end{tabular}

Note: CMSS, the Cox Menstrual Symptom Scale; FMA, Fugl - Meyer motor assessment scale; MBI, modified Barthel index; TCM, traditional Chinese medicine; MACE, major adverse cardiovascular events; HFC, changes in heart function classification; LVEF, left ventricular ejection fraction; AAS, auricular acupoint stimulation; WA, warming acupuncture; ACE, acupoint catgut embedding; EA, electroacupuncture; AR, acupuncture and related therapies.

TABLE 3: Methodological quality assessment of the included NMAs.

\begin{tabular}{|c|c|c|c|c|c|c|c|c|c|c|c|c|c|c|c|c|c|}
\hline Item & 1 & 2 & 3 & 4 & 5 & 6 & 7 & 8 & 9 & 10 & 11 & 12 & 13 & 14 & 15 & 16 & Score \\
\hline Fu et al. [15] & $\mathrm{Y}$ & $\mathrm{N}$ & $\mathrm{N}$ & $\mathrm{P}$ & $\mathrm{Y}$ & $\mathrm{Y}$ & $\mathrm{N}$ & $P$ & $\mathrm{P}$ & $\mathrm{N}$ & $\mathrm{N}$ & $\mathrm{N}$ & $\mathrm{N}$ & $\mathrm{N}$ & $\mathrm{Y}$ & $\mathrm{N}$ & 5.5 \\
\hline Ding [16] & $\mathrm{Y}$ & $\mathrm{N}$ & $\mathrm{N}$ & $\mathrm{P}$ & $\mathrm{Y}$ & $\mathrm{N}$ & $\mathrm{N}$ & $\mathrm{Y}$ & Y & $\mathrm{N}$ & $\mathrm{N}$ & $\mathrm{N}$ & $\mathrm{N}$ & $\mathrm{N}$ & $\mathrm{N}$ & $\mathrm{P}$ & 5 \\
\hline $\mathrm{Li}[17]$ & $\mathrm{Y}$ & $\mathrm{N}$ & $\mathrm{N}$ & $\mathrm{P}$ & $\mathrm{Y}$ & $\mathrm{N}$ & $\mathrm{N}$ & $\mathrm{P}$ & $\mathrm{Y}$ & $\mathrm{N}$ & $\mathrm{Y}$ & $\mathrm{N}$ & $\mathrm{P}$ & $\mathrm{N}$ & $\mathrm{N}$ & $\mathrm{N}$ & 5.5 \\
\hline $\mathrm{Bu}$ et al. [18] & $\mathrm{Y}$ & $\mathrm{N}$ & $\mathrm{N}$ & $\mathrm{Y}$ & $\mathrm{Y}$ & $\mathrm{Y}$ & $\mathrm{N}$ & $\mathrm{P}$ & $\mathrm{Y}$ & $\mathrm{N}$ & $\mathrm{P}$ & $\mathrm{N}$ & $\mathrm{P}$ & $\mathrm{N}$ & $\mathrm{N}$ & $\mathrm{P}$ & 7 \\
\hline Zhang [19] & $\mathrm{Y}$ & $\mathrm{N}$ & $\mathrm{N}$ & $\mathrm{Y}$ & $\mathrm{N}$ & $\mathrm{Y}$ & $\mathrm{N}$ & $\mathrm{P}$ & $\mathrm{Y}$ & $\mathrm{N}$ & $\mathrm{Y}$ & $\mathrm{N}$ & $\mathrm{P}$ & $\mathrm{Y}$ & $\mathrm{N}$ & $\mathrm{P}$ & 6 \\
\hline Song [20] & $\mathrm{Y}$ & $\mathrm{N}$ & $\mathrm{N}$ & $\mathrm{Y}$ & $\mathrm{Y}$ & $\mathrm{Y}$ & $\mathrm{N}$ & $\mathrm{Y}$ & $\mathrm{Y}$ & $\mathrm{N}$ & $\mathrm{P}$ & $\mathrm{P}$ & $\mathrm{P}$ & $\mathrm{Y}$ & $\mathrm{Y}$ & $\mathrm{P}$ & 8 \\
\hline Yang et al. [21] & $\mathrm{Y}$ & $\mathrm{N}$ & $\mathrm{N}$ & $\mathrm{P}$ & $\mathrm{N}$ & $\mathrm{Y}$ & $\mathrm{N}$ & $\mathrm{Y}$ & $\mathrm{Y}$ & $\mathrm{N}$ & $\mathrm{Y}$ & $\mathrm{N}$ & $\mathrm{Y}$ & $\mathrm{Y}$ & $\mathrm{N}$ & $\mathrm{P}$ & 8 \\
\hline $\mathrm{Li}[22]$ & $\mathrm{Y}$ & $\mathrm{N}$ & $\mathrm{N}$ & $\mathrm{Y}$ & $\mathrm{Y}$ & $\mathrm{Y}$ & $\mathrm{N}$ & $\mathrm{Y}$ & $\mathrm{Y}$ & $\mathrm{N}$ & $\mathrm{Y}$ & $\mathrm{Y}$ & $\mathrm{Y}$ & $\mathrm{Y}$ & $\mathrm{P}$ & $\mathrm{P}$ & 11 \\
\hline Jia et al. [23] & $\mathrm{Y}$ & $\mathrm{N}$ & $\mathrm{N}$ & $\mathrm{P}$ & $\mathrm{Y}$ & $\mathrm{N}$ & $\mathrm{N}$ & $\mathrm{Y}$ & $\mathrm{Y}$ & $\mathrm{N}$ & $\mathrm{Y}$ & $\mathrm{Y}$ & $\mathrm{Y}$ & $\mathrm{Y}$ & $\mathrm{P}$ & $\mathrm{P}$ & 9.5 \\
\hline Liu [24] & $\mathrm{Y}$ & $\mathrm{N}$ & $\mathrm{N}$ & $\mathrm{Y}$ & $\mathrm{Y}$ & $\mathrm{Y}$ & $\mathrm{N}$ & $\mathrm{Y}$ & $\mathrm{Y}$ & $\mathrm{N}$ & $\mathrm{Y}$ & $\mathrm{Y}$ & $\mathrm{N}$ & $\mathrm{Y}$ & $\mathrm{N}$ & $\mathrm{P}$ & 9.5 \\
\hline Feng [25] & $\mathrm{Y}$ & $\mathrm{N}$ & $\mathrm{N}$ & $\mathrm{Y}$ & $\mathrm{P}$ & $\mathrm{Y}$ & $\mathrm{N}$ & $\mathrm{Y}$ & Y & $\mathrm{N}$ & $\mathrm{Y}$ & $\mathrm{Y}$ & $\mathrm{Y}$ & $\mathrm{Y}$ & $\mathrm{P}$ & $\mathrm{N}$ & 10 \\
\hline Liu $[26]$ & $\mathrm{Y}$ & $\mathrm{N}$ & $\mathrm{N}$ & $\mathrm{P}$ & $\mathrm{N}$ & $\mathrm{N}$ & $\mathrm{N}$ & $\mathrm{Y}$ & $\mathrm{Y}$ & $\mathrm{N}$ & $\mathrm{Y}$ & $\mathrm{N}$ & $\mathrm{P}$ & $\mathrm{N}$ & $\mathrm{N}$ & $\mathrm{P}$ & 5.5 \\
\hline Yang et al. [27] & $\mathrm{Y}$ & $\mathrm{N}$ & $\mathrm{N}$ & $\mathrm{Y}$ & $\mathrm{Y}$ & $\mathrm{Y}$ & $\mathrm{N}$ & $\mathrm{Y}$ & $\mathrm{Y}$ & $\mathrm{N}$ & $\mathrm{Y}$ & $\mathrm{Y}$ & $\mathrm{Y}$ & $\mathrm{Y}$ & $\mathrm{Y}$ & $\mathrm{P}$ & 11 \\
\hline Zhu et al. [28] & $\mathrm{N}$ & $\mathrm{N}$ & $\mathrm{N}$ & $\mathrm{Y}$ & $\mathrm{Y}$ & $\mathrm{Y}$ & $\mathrm{N}$ & $\mathrm{N}$ & $\mathrm{Y}$ & $\mathrm{N}$ & $\mathrm{Y}$ & $\mathrm{N}$ & $\mathrm{N}$ & $\mathrm{N}$ & $\mathrm{Y}$ & $\mathrm{P}$ & 6.5 \\
\hline Zheng [29] & $\mathrm{P}$ & $\mathrm{Y}$ & $\mathrm{Y}$ & $\mathrm{Y}$ & $\mathrm{Y}$ & $\mathrm{Y}$ & $\mathrm{N}$ & $\mathrm{P}$ & $\mathrm{Y}$ & $\mathrm{N}$ & $\mathrm{Y}$ & $\mathrm{N}$ & $\mathrm{Y}$ & $\mathrm{Y}$ & $\mathrm{P}$ & $\mathrm{Y}$ & 11 \\
\hline Qin [30] & $\mathrm{Y}$ & $\mathrm{N}$ & $\mathrm{N}$ & $\mathrm{Y}$ & $\mathrm{Y}$ & $\mathrm{Y}$ & $\mathrm{N}$ & $\mathrm{Y}$ & $\mathrm{Y}$ & $\mathrm{N}$ & $\mathrm{Y}$ & $\mathrm{Y}$ & $\mathrm{Y}$ & $\mathrm{Y}$ & $\mathrm{N}$ & $\mathrm{Y}$ & 11 \\
\hline Li et al. [31] & $\mathrm{Y}$ & $\mathrm{N}$ & $\mathrm{N}$ & $\mathrm{P}$ & $\mathrm{Y}$ & $\mathrm{Y}$ & $\mathrm{N}$ & $\mathrm{P}$ & $\mathrm{Y}$ & $\mathrm{N}$ & $\mathrm{Y}$ & $\mathrm{Y}$ & $\mathrm{Y}$ & $\mathrm{Y}$ & $\mathrm{Y}$ & $\mathrm{Y}$ & 11 \\
\hline Mo et al. [32] & $\mathrm{P}$ & $\mathrm{N}$ & $\mathrm{N}$ & $\mathrm{Y}$ & $\mathrm{Y}$ & $\mathrm{Y}$ & $\mathrm{N}$ & $\mathrm{P}$ & $\mathrm{Y}$ & $\mathrm{N}$ & $\mathrm{P}$ & $\mathrm{N}$ & $\mathrm{N}$ & $\mathrm{N}$ & $\mathrm{Y}$ & $\mathrm{Y}$ & 7.5 \\
\hline Luo et al. [33] & $\mathrm{Y}$ & $\mathrm{N}$ & $\mathrm{N}$ & $\mathrm{P}$ & $\mathrm{N}$ & $\mathrm{N}$ & $\mathrm{N}$ & $\mathrm{P}$ & $\mathrm{Y}$ & $\mathrm{N}$ & $\mathrm{Y}$ & $\mathrm{Y}$ & $\mathrm{N}$ & $\mathrm{N}$ & $\mathrm{Y}$ & $\mathrm{P}$ & 6.5 \\
\hline Yeh et al. [34] & $\mathrm{P}$ & $\mathrm{N}$ & $\mathrm{N}$ & $\mathrm{P}$ & $\mathrm{Y}$ & $\mathrm{Y}$ & $\mathrm{N}$ & $\mathrm{P}$ & $\mathrm{Y}$ & $\mathrm{N}$ & $\mathrm{Y}$ & $\mathrm{Y}$ & $\mathrm{Y}$ & $\mathrm{Y}$ & $\mathrm{Y}$ & $\mathrm{P}$ & 10 \\
\hline Chen et al. [35] & $\mathrm{P}$ & $\mathrm{Y}$ & $\mathrm{N}$ & $\mathrm{Y}$ & $\mathrm{Y}$ & $\mathrm{N}$ & $\mathrm{N}$ & $\mathrm{P}$ & $\mathrm{Y}$ & $\mathrm{N}$ & $\mathrm{Y}$ & $\mathrm{N}$ & $\mathrm{N}$ & $\mathrm{P}$ & $\mathrm{N}$ & $\mathrm{Y}$ & 7.5 \\
\hline Tan et al. [36] & $\mathrm{Y}$ & $\mathrm{N}$ & $\mathrm{N}$ & $\mathrm{P}$ & $\mathrm{Y}$ & $\mathrm{Y}$ & $\mathrm{N}$ & $\mathrm{Y}$ & $\mathrm{Y}$ & $\mathrm{N}$ & $\mathrm{Y}$ & $\mathrm{Y}$ & $\mathrm{Y}$ & $\mathrm{N}$ & $\mathrm{Y}$ & $\mathrm{Y}$ & 10.5 \\
\hline Li et al. [37] & $\mathrm{Y}$ & $\mathrm{Y}$ & $\mathrm{N}$ & $\mathrm{Y}$ & $\mathrm{Y}$ & $\mathrm{Y}$ & $\mathrm{N}$ & $\mathrm{Y}$ & $\mathrm{Y}$ & $\mathrm{N}$ & $\mathrm{Y}$ & $\mathrm{Y}$ & $\mathrm{Y}$ & $\mathrm{N}$ & $\mathrm{N}$ & $\mathrm{P}$ & 10.5 \\
\hline Zhu et al. [38] & $\mathrm{P}$ & $\mathrm{N}$ & $\mathrm{N}$ & $\mathrm{Y}$ & $\mathrm{Y}$ & $\mathrm{Y}$ & $\mathrm{N}$ & $\mathrm{P}$ & $\mathrm{Y}$ & $\mathrm{N}$ & $\mathrm{Y}$ & $\mathrm{Y}$ & $\mathrm{Y}$ & $\mathrm{P}$ & $\mathrm{Y}$ & $\mathrm{P}$ & 10 \\
\hline Xiong and Chen [39] & $\mathrm{P}$ & $\mathrm{N}$ & $\mathrm{N}$ & $\mathrm{P}$ & $\mathrm{N}$ & $\mathrm{N}$ & $\mathrm{N}$ & $\mathrm{P}$ & $\mathrm{N}$ & $\mathrm{N}$ & $\mathrm{N}$ & $\mathrm{N}$ & $\mathrm{N}$ & $\mathrm{N}$ & $\mathrm{N}$ & $\mathrm{Y}$ & 2.5 \\
\hline Yang et al. [40] & $\mathrm{Y}$ & $\mathrm{Y}$ & $\mathrm{N}$ & $\mathrm{P}$ & $\mathrm{Y}$ & $\mathrm{Y}$ & $\mathrm{N}$ & $\mathrm{P}$ & $\mathrm{Y}$ & $\mathrm{N}$ & $\mathrm{Y}$ & $\mathrm{Y}$ & $\mathrm{Y}$ & $\mathrm{N}$ & $\mathrm{Y}$ & $\mathrm{Y}$ & 11 \\
\hline
\end{tabular}


TABLE 3: Continued.

\begin{tabular}{lccccccccccccccccc}
\hline Item & 1 & 2 & 3 & 4 & 5 & 6 & 7 & 8 & 9 & 10 & 11 & 12 & 13 & 14 & 15 & 16 & Score \\
\hline Zhang et al. [41] & $\mathrm{Y}$ & $\mathrm{N}$ & $\mathrm{N}$ & $\mathrm{P}$ & $\mathrm{Y}$ & $\mathrm{Y}$ & $\mathrm{N}$ & $\mathrm{Y}$ & $\mathrm{Y}$ & $\mathrm{N}$ & $\mathrm{Y}$ & $\mathrm{Y}$ & $\mathrm{Y}$ & $\mathrm{N}$ & $\mathrm{N}$ & $\mathrm{Y}$ & 9.5 \\
Corbett 2013 [42] & $\mathrm{Y}$ & $\mathrm{Y}$ & $\mathrm{Y}$ & $\mathrm{Y}$ & $\mathrm{Y}$ & $\mathrm{Y}$ & $\mathrm{N}$ & $\mathrm{P}$ & $\mathrm{Y}$ & $\mathrm{N}$ & $\mathrm{Y}$ & $\mathrm{Y}$ & $\mathrm{Y}$ & $\mathrm{Y}$ & $\mathrm{Y}$ & $\mathrm{Y}$ & 13.5 \\
Wang et al. [43] & $\mathrm{Y}$ & $\mathrm{N}$ & $\mathrm{N}$ & $\mathrm{P}$ & $\mathrm{N}$ & $\mathrm{Y}$ & $\mathrm{N}$ & $\mathrm{Y}$ & $\mathrm{Y}$ & $\mathrm{N}$ & $\mathrm{Y}$ & $\mathrm{Y}$ & $\mathrm{Y}$ & $\mathrm{Y}$ & $\mathrm{Y}$ & $\mathrm{Y}$ & 10.5 \\
Score & 20 & 5 & 2 & 15 & 22 & 22 & 0 & 14 & 27 & 0 & 23 & 16 & 16 & 14 & 13 & 11 & $8.64 / 9.5$ \\
\hline
\end{tabular}

$\mathrm{Y}$, yes $(1$ point); $\mathrm{N}$, no $(0$ point); $\mathrm{P}$, partial satisfaction $(0.5$ point). Item 1 , did the research questions and inclusion criteria for the review include the components of PICO? Item 2, did the report of the review contain an explicit statement that the review methods were established prior to the conduct of the review and did the report justify any significant deviations from the protocol? Item 3, did the review authors explain their selection of the study designs for inclusion in the review? Item 4, did the review authors use a comprehensive literature search strategy? Item 5 , did the review authors perform study selection in duplicate? Item 6, did the review authors perform data extraction in duplicate? Item 7, did the review authors provide a list of excluded studies and justify the exclusions? Item 8, did the review authors describe the included studies in adequate detail? Item 9, did the review authors use a satisfactory technique for assessing the risk of bias (RoB) in individual studies that were included in the review? Item 10, did the review authors report on the sources of funding for the studies included in the review? Item 11, if meta-analysis was performed, did the review authors use appropriate methods for statistical combination of results? Item 12, if meta-analysis was performed, did the review authors assess the potential impact of RoB in individual studies on the results of the meta-analysis or other evidence synthesis? Item 13, did the review authors account for RoB in individual studies when interpreting/ discussing the results of the review? Item 14, did the review authors provide a satisfactory explanation for, and discussion of, any heterogeneity observed in the results of the review? Item 15, if they performed quantitative synthesis, did the review authors carry out an adequate investigation of publication bias (small study bias) and discuss its likely impact on the results of the review? Item 16, did the review authors report any potential sources of conflict of interest, including any funding they received for conducting the review?

TABLE 4: Summary of methodological quality assessment.

\begin{tabular}{|c|c|c|c|c|c|c|c|}
\hline \multirow[b]{2}{*}{ Number } & \multirow[b]{2}{*}{ Item } & \multicolumn{2}{|c|}{ Completely reported } & \multicolumn{2}{|c|}{ Partially reported } & \multicolumn{2}{|c|}{ Not reported } \\
\hline & & $\begin{array}{c}\text { Frequency } \\
(\%)\end{array}$ & $95 \% \mathrm{CI}$ & $\begin{array}{c}\text { Frequency } \\
(\%)\end{array}$ & $95 \% \mathrm{CI}$ & $\begin{array}{c}\text { Frequency } \\
(\%)\end{array}$ & $95 \% \mathrm{CI}$ \\
\hline 1 & Components of PICO question? & $22(75.86 \%)$ & $(0.58,0.94)$ & $6(20.69 \%)$ & $(-0.12,0.53)$ & $1(3.45 \%)$ & $(-0.32,0.39)$ \\
\hline 2 & Review protocol? & $5(17.24 \%)$ & $\begin{array}{l}(-0.16 \\
0.50)\end{array}$ & 0 & & $24(82.75 \%)$ & $(0.68,0.98)$ \\
\hline 3 & Explanation of study design? & $2(6.90 \%)$ & $\begin{array}{c}(-0.28 \\
0.42)\end{array}$ & 0 & & $27(93.10 \%)$ & $(0.84,1.03)$ \\
\hline 4 & $\begin{array}{c}\text { Comprehensive literature search } \\
\text { strategy }\end{array}$ & $16(55.17 \%)$ & $(0.31,0.80)$ & $13(44.83 \%)$ & $(0.18,0.72)$ & 0 & \\
\hline 5 & Study selection in duplicate? & $22(75.86 \%)$ & $(0.58,0.94)$ & $1(3.45 \%)$ & $(-0.32,0.39)$ & $6(20.69 \%)$ & $(-0.12,0.53)$ \\
\hline 6 & Data extraction in duplicate? & $22(75.86 \%)$ & $(0.58,0.94)$ & 0 & & $7(24.14 \%)$ & $(-0.08,0.56)$ \\
\hline 7 & $\begin{array}{l}\text { List of excluded studies and justify the } \\
\text { exclusions? }\end{array}$ & 0 & & 0 & & $29(100 \%)$ & \\
\hline 8 & Study characteristics & $14(48.27 \%)$ & $(0.22,0.74)$ & $14(48.27 \%)$ & $(0.22,0.74)$ & $1(3.45 \%)$ & $(-0.32,0.39)$ \\
\hline 9 & $\begin{array}{l}\text { Satisfactory technique for assessing risk } \\
\text { of bias? }\end{array}$ & $27(93.10 \%)$ & $(0.84,1.03)$ & $1(3.45 \%)$ & $(-0.32,0.39)$ & $1(3.45 \%)$ & $(-0.32,0.39)$ \\
\hline 10 & Sources of funding for each study? & 0 & & 0 & & $29(100 \%)$ & \\
\hline 11 & Appropriate methods? & $23(79.31 \%)$ & $(0.63,0.96)$ & $3(10.34 \%)$ & $(-0.24,0.45)$ & $3(10.34 \%)$ & $(-0.24,0.45)$ \\
\hline 12 & $\begin{array}{l}\text { Assess potential impact of risk of bias on } \\
\text { the results? }\end{array}$ & $16(55.17 \%)$ & $(0.31,0.80)$ & $1(3.45 \%)$ & $(-0.32,0.39)$ & $12(41.38 \%)$ & $(0.14,0.69)$ \\
\hline 13 & $\begin{array}{l}\text { Account for risk of bias when } \\
\text { interpreting/discussing? }\end{array}$ & $16(55.17 \%)$ & $(0.31,0.80)$ & $5(17.24 \%)$ & $(-0.16,0.50)$ & $8(27.59 \%)$ & $(-0.03,0.59)$ \\
\hline 14 & $\begin{array}{l}\text { Satisfactory explanation for and } \\
\text { discussion of any heterogeneity? }\end{array}$ & $14(48.27 \%)$ & $(0.29,0.81)$ & $2(6.90 \%)$ & $(-0.28,0.42)$ & $13(44.83 \%)$ & $(0.18,0.72)$ \\
\hline 15 & $\begin{array}{l}\text { Publication bias (small sample bias) } \\
\text { assessed and discussed? }\end{array}$ & $13(44.83 \%)$ & $(0.18,0.72)$ & $4(13.79 \%)$ & $(-0.20,0.48)$ & $12(41.38 \%)$ & $(0.14,0.69)$ \\
\hline 16 & $\begin{array}{l}\text { Publication bias (small sample bias) } \\
\text { assessed and discussed? }\end{array}$ & $11(37.93 \%)$ & $(0.09,0.67)$ & $15(51.72 \%)$ & $(0.26,0.77)$ & $3(10.34 \%)$ & $(-0.24,0.45)$ \\
\hline
\end{tabular}

This research also had presented some limitations: first, there was no specific methodological quality assessment tool for NMAs, even though AMSTAR2 was generally used in the quality evaluation of systematic review and meta-analysis.
Second, in this study, the quality of each NMA was quantified by the assignment method, and there were some controversies on whether the weight of each item was consistent. Third, even if a comprehensive literature search strategy was used, there 


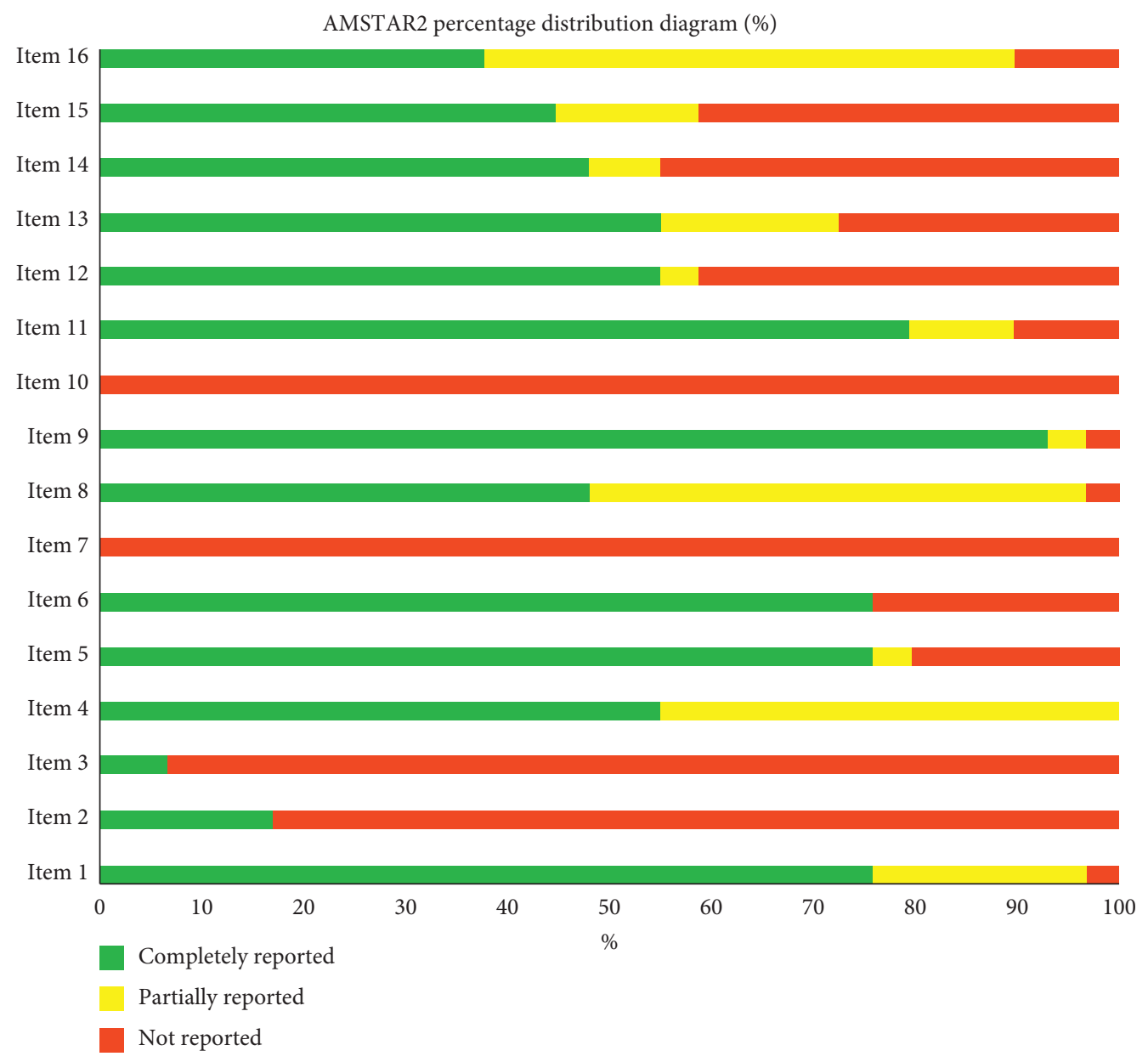

FIGURE 2: AMSTAR2 percentage distribution diagram (\%).

TABle 5: Reporting quality assessment of the included NMAs.

\begin{tabular}{|c|c|c|c|c|c|c|c|c|c|c|c|c|c|c|c|c|}
\hline Item & Section/topic & $\begin{array}{c}\mathrm{Fu} \\
2019\end{array}$ & $\begin{array}{l}\text { Ding } \\
2019 \\
\end{array}$ & $\begin{array}{c}\mathrm{Li} \\
2018 \\
\end{array}$ & $\begin{array}{c}\mathrm{Bu} \\
2017 \\
\end{array}$ & $\begin{array}{c}\text { Zhang } \\
2018\end{array}$ & $\begin{array}{l}\text { Song } \\
2019 \\
\end{array}$ & $\begin{array}{l}\text { Yang } \\
2016 \\
\end{array}$ & $\begin{array}{c}\mathrm{Li} \\
2017 \\
\end{array}$ & $\begin{array}{c}\text { Jia } \\
2018 \\
\end{array}$ & $\begin{array}{c}\text { Liu } \\
2016 \\
\end{array}$ & $\begin{array}{l}\text { Feng } \\
2018 \\
\end{array}$ & $\begin{array}{c}\text { Liu } \\
2019 \\
\end{array}$ & $\begin{array}{l}\text { Yang } \\
2017 \\
\end{array}$ & $\begin{array}{l}\text { Zhu } \\
2018 \\
\end{array}$ & $\begin{array}{c}\text { Zheng } \\
2018 \\
\end{array}$ \\
\hline 1 & Title & 1 & 1 & 1 & 1 & 1 & 1 & 1 & 1 & 1 & 1 & 1 & 1 & 1 & 1 & 1 \\
\hline 2 & $\begin{array}{l}\text { Structured } \\
\text { summary }\end{array}$ & 0.5 & 0.5 & 0.5 & 0.5 & 0.5 & 0.5 & 0.5 & 0.5 & 0.5 & 0.5 & 0.5 & 0.5 & 0.5 & 0.5 & 1 \\
\hline 3 & Rationale & 1 & 0.5 & 1 & 0.5 & 1 & 1 & 1 & 1 & 1 & 1 & 0.5 & 1 & 1 & 1 & 1 \\
\hline 4 & Objectives & 1 & 1 & 1 & 0.5 & 1 & 1 & 1 & 1 & 1 & 1 & 1 & 1 & 1 & 1 & 1 \\
\hline 5 & $\begin{array}{l}\text { Protocol and } \\
\text { registration }\end{array}$ & 0 & 0 & 0 & 0 & 0 & 0 & 0 & 0 & 0 & 0 & 0 & 0 & 0 & 0 & 1 \\
\hline 6 & $\begin{array}{c}\text { Eligibility } \\
\text { criteria }\end{array}$ & 1 & 1 & 0.5 & 0.5 & 0.5 & 1 & 1 & 1 & 1 & 1 & 1 & 1 & 1 & 1 & 0.5 \\
\hline 7 & $\begin{array}{l}\text { Information } \\
\text { sources }\end{array}$ & 1 & 1 & 1 & 1 & 1 & 1 & 0.5 & 1 & 1 & 1 & 1 & 1 & 1 & 1 & 1 \\
\hline 8 & Search & 0 & 0 & 0 & 0 & 0 & 0 & 0 & 1 & 0 & 1 & 1 & 0 & 1 & 0 & 1 \\
\hline 9 & $\begin{array}{c}\text { Study } \\
\text { selection } \\
\text { Data }\end{array}$ & 0.5 & 1 & 1 & 1 & 0 & 1 & 0 & 1 & 1 & 1 & 1 & 0 & 1 & 1 & 1 \\
\hline 10 & $\begin{array}{l}\text { collection } \\
\text { process }\end{array}$ & 0.5 & 0.5 & 0.5 & 1 & 0.5 & 0.5 & 0.5 & 1 & 0.5 & 0.5 & 1 & 0.5 & 0.5 & 0.5 & 0.5 \\
\hline 11 & Data items & 0.5 & 0.5 & 1 & 0.5 & 0.5 & 0.5 & 0.5 & 0.5 & 0.5 & 0.5 & 0.5 & 0.5 & 0.5 & 0.5 & 0.5 \\
\hline S1 & $\begin{array}{l}\text { Geometry of } \\
\text { the network }\end{array}$ & 1 & 0 & 1 & 0 & 1 & 1 & 0 & 1 & 1 & 1 & 1 & 1 & 1 & 1 & 1 \\
\hline
\end{tabular}


TABle 5: Continued.

\begin{tabular}{|c|c|c|c|c|c|c|c|c|c|c|c|c|c|c|c|c|}
\hline Item & Section/topic & $\begin{array}{c}\mathrm{Fu} \\
2019\end{array}$ & $\begin{array}{l}\text { Ding } \\
2019 \\
\end{array}$ & $\begin{array}{c}\mathrm{Li} \\
2018\end{array}$ & $\begin{array}{c}\mathrm{Bu} \\
2017\end{array}$ & $\begin{array}{c}\text { Zhang } \\
2018\end{array}$ & $\begin{array}{l}\text { Song } \\
2019\end{array}$ & $\begin{array}{l}\text { Yang } \\
2016\end{array}$ & $\begin{array}{c}\mathrm{Li} \\
2017\end{array}$ & $\begin{array}{c}\text { Jia } \\
2018\end{array}$ & $\begin{array}{c}\text { Liu } \\
2016\end{array}$ & $\begin{array}{l}\text { Feng } \\
2018 \\
\end{array}$ & $\begin{array}{c}\text { Liu } \\
2019\end{array}$ & $\begin{array}{l}\text { Yang } \\
2017\end{array}$ & $\begin{array}{l}\text { Zhu } \\
2018\end{array}$ & $\begin{array}{c}\text { Zheng } \\
2018 \\
\end{array}$ \\
\hline 12 & $\begin{array}{l}\text { Risk of bias } \\
\text { within } \\
\text { individual } \\
\text { studies }\end{array}$ & 1 & 1 & 1 & 1 & 1 & 1 & 1 & 1 & 1 & 1 & 1 & 1 & 1 & 1 & 1 \\
\hline 13 & $\begin{array}{c}\text { Summary } \\
\text { measures }\end{array}$ & 1 & 0.5 & 1 & 0.5 & 0.5 & 0.5 & 0.5 & 1 & 1 & 1 & 1 & 1 & 1 & 1 & 1 \\
\hline 14 & $\begin{array}{l}\text { Planned } \\
\text { methods of } \\
\text { analysis }\end{array}$ & 1 & 0 & 1 & 1 & 0 & 1 & 1 & 1 & 1 & 1 & 1 & 1 & 1 & 1 & 1 \\
\hline S2 & $\begin{array}{l}\text { Assessment of } \\
\text { inconsistency }\end{array}$ & 1 & 0 & 1 & 1 & 1 & 1 & 1 & 1 & 1 & 0 & 1 & 1 & 1 & 1 & 1 \\
\hline 15 & $\begin{array}{l}\text { Risk of bias } \\
\text { across studies }\end{array}$ & 1 & 0 & 0 & 1 & 0 & 1 & 0 & 1 & 1 & 1 & 1 & 0 & 1 & 1 & 1 \\
\hline 16 & $\begin{array}{c}\text { Additional } \\
\text { analyses }\end{array}$ & 0 & 0 & 0 & 0 & 0 & 1 & 0 & 0 & 1 & 1 & 1 & 0 & 0 & 1 & 1 \\
\hline 17 & $\begin{array}{c}\text { Study } \\
\text { selection }\end{array}$ & 1 & 1 & 0.5 & 1 & 0.5 & 0.5 & 1 & 1 & 1 & 1 & 1 & 1 & 1 & 1 & 1 \\
\hline S3 & $\begin{array}{l}\text { Presentation } \\
\text { of network } \\
\text { structure }\end{array}$ & 1 & 1 & 1 & 1 & 1 & 1 & 0 & 1 & 1 & 1 & 1 & 1 & 1 & 1 & 1 \\
\hline S4 & $\begin{array}{c}\text { Summary of } \\
\text { network } \\
\text { geometry }\end{array}$ & 1 & 0.5 & 0.5 & 0.5 & 0.5 & 0.5 & 0 & 0.5 & 0.5 & 0.5 & 0.5 & 0.5 & 0.5 & 0 & 0.5 \\
\hline 18 & $\begin{array}{c}\text { Study } \\
\text { characteristics }\end{array}$ & 1 & 1 & 1 & 1 & 1 & 1 & 1 & 1 & 1 & 1 & 1 & 1 & 1 & 1 & 1 \\
\hline 19 & $\begin{array}{l}\text { Risk of bias } \\
\text { within studies }\end{array}$ & 1 & 1 & 1 & 1 & 1 & 1 & 1 & 1 & 1 & 1 & 1 & 1 & 1 & 1 & 0 \\
\hline 20 & $\begin{array}{c}\text { Results of } \\
\text { individual } \\
\text { studies }\end{array}$ & 0 & 0 & 0 & 0 & 0 & 0 & 0 & 1 & 1 & 0 & 1 & 0 & 1 & 1 & 0 \\
\hline 21 & $\begin{array}{l}\text { Synthesis of } \\
\text { results }\end{array}$ & 1 & 1 & 1 & 1 & 1 & 0.5 & 1 & 1 & 1 & 1 & 1 & 1 & 1 & 1 & 1 \\
\hline S5 & $\begin{array}{l}\text { Exploration } \\
\text { for } \\
\text { inconsistency }\end{array}$ & 1 & 0 & 0 & 1 & 1 & 1 & 1 & 1 & 1 & 1 & 1 & 1 & 0 & 0 & 0 \\
\hline 22 & $\begin{array}{l}\text { Risk of bias } \\
\text { across studies }\end{array}$ & 1 & 0 & 0 & 0 & 0 & 1 & 0 & 1 & 1 & 0 & 1 & 0 & 0 & 1 & 0 \\
\hline 23 & $\begin{array}{c}\text { Results of } \\
\text { additional } \\
\text { analyses }\end{array}$ & 0 & 0 & 0 & 0 & 1 & 0 & 0 & 0 & 0 & 1 & 0 & 0 & 0 & 1 & 0 \\
\hline 24 & $\begin{array}{c}\text { Summary of } \\
\text { evidence }\end{array}$ & 1 & 0 & 1 & 0 & 0.5 & 1 & 1 & 1 & 1 & 1 & 1 & 1 & 1 & 1 & 1 \\
\hline 25 & Limitations & 1 & 1 & 1 & 1 & 1 & 1 & 1 & 1 & 1 & 1 & 1 & 1 & 1 & 1 & 1 \\
\hline 26 & Conclusions & 1 & 1 & 0.5 & 1 & 1 & 0.5 & 1 & 1 & 1 & 1 & 1 & 1 & 1 & 1 & 1 \\
\hline 27 & Funding & 0 & 1 & 0 & 1 & 1 & 1 & 1 & 1 & 0 & 1 & 0 & 1 & 1 & 0 & 1 \\
\hline & Summary & 24 & 17 & 20 & 20.5 & 20 & 24 & 18.5 & 27.5 & 26 & 26 & 27 & 22 & 25 & 25.5 & 25 \\
\hline Item & & $\begin{array}{c}\text { Qin } \\
2016 \\
\end{array}$ & $\begin{array}{c}\mathrm{Li} \\
2018 \\
\end{array}$ & $\begin{array}{c}\text { Mo } \\
2019 \\
\end{array}$ & $\begin{array}{l}\text { Luo } \\
2018 \\
\end{array}$ & $\begin{array}{c}\text { Yeh } \\
2016\end{array}$ & $\begin{array}{l}\text { Chen } \\
2019 \\
\end{array}$ & $\begin{array}{c}\text { Tan } \\
2019 \\
\end{array}$ & $\begin{array}{c}\mathrm{Li} \\
2017\end{array}$ & $\begin{array}{l}\text { Zhu } \\
2018\end{array}$ & $\begin{array}{c}\text { Xiong } \\
2018 \\
\end{array}$ & $\begin{array}{l}\text { Yang } \\
2019 \\
\end{array}$ & $\begin{array}{c}\text { Zhang } \\
2018\end{array}$ & $\begin{array}{c}\text { S. } \\
2013 \\
\end{array}$ & $\begin{array}{l}\text { Wang } \\
2017\end{array}$ & \\
\hline 1 & & 1 & 1 & 1 & 1 & 1 & 1 & 1 & 1 & 1 & 1 & 1 & 1 & 1 & 1 & \\
\hline 2 & & 0.5 & 0.5 & 0.5 & 0.5 & 0.5 & 1 & 0.5 & 1 & 0.5 & 0.5 & 1 & 0.5 & 1 & 0.5 & \\
\hline 3 & & 1 & 1 & 1 & 1 & 1 & 1 & 1 & 1 & 1 & 1 & 1 & 1 & 1 & 1 & \\
\hline 4 & & 1 & 1 & 1 & 1 & 1 & 1 & 1 & 1 & 1 & 1 & 1 & 1 & 1 & 1 & \\
\hline 5 & & 0 & 0 & 0 & 0 & 0 & 1 & 0 & 1 & 0 & 0 & 1 & 0 & 1 & 0 & \\
\hline 6 & & 1 & 1 & 0.5 & 1 & 0.5 & 0.5 & 1 & 1 & 0.5 & 0.5 & 1 & 1 & 1 & 0.5 & \\
\hline 7 & & 1 & 0.5 & 1 & 1 & 0.5 & 1 & 1 & 1 & 1 & 0.5 & 0.5 & 1 & 1 & 1 & \\
\hline 8 & & 1 & 0 & 1 & 0 & 0 & 0 & 1 & 1 & 1 & 0 & 1 & 1 & 0 & 0 & \\
\hline 9 & & 1 & 1 & 1 & 0.5 & 1 & 1 & 1 & 1 & 1 & 0 & 1 & 1 & 1 & 0 & \\
\hline 10 & & 1 & 0.5 & 0.5 & 0.5 & 0.5 & 0.5 & 0.5 & 0.5 & 0.5 & 0 & 0.5 & 1 & 0.5 & 0.5 & \\
\hline 11 & & 1 & 0.5 & 0.5 & 0.5 & 0.5 & 0.5 & 1 & 0.5 & 0.5 & 0.5 & 0.5 & 0.5 & 0.5 & 0.5 & \\
\hline
\end{tabular}


TABle 5: Continued.

\begin{tabular}{|c|c|c|c|c|c|c|c|c|c|c|c|c|c|c|c|c|}
\hline Item & Section/topic & $\begin{array}{c}\mathrm{Fu} \\
2019 \\
\end{array}$ & $\begin{array}{l}\text { Ding } \\
2019 \\
\end{array}$ & $\begin{array}{c}\mathrm{Li} \\
2018 \\
\end{array}$ & $\begin{array}{c}\mathrm{Bu} \\
2017\end{array}$ & $\begin{array}{c}\text { Zhang } \\
2018 \\
\end{array}$ & $\begin{array}{l}\text { Song } \\
2019 \\
\end{array}$ & $\begin{array}{l}\text { Yang } \\
2016 \\
\end{array}$ & $\begin{array}{c}\mathrm{Li} \\
2017 \\
\end{array}$ & $\begin{array}{c}\text { Jia } \\
2018 \\
\end{array}$ & $\begin{array}{c}\text { Liu } \\
2016 \\
\end{array}$ & $\begin{array}{l}\text { Feng } \\
2018 \\
\end{array}$ & $\begin{array}{c}\text { Liu } \\
2019 \\
\end{array}$ & $\begin{array}{l}\text { Yang } \\
2017 \\
\end{array}$ & $\begin{array}{l}\text { Zhu } \\
2018 \\
\end{array}$ & $\begin{array}{c}\text { Zheng } \\
2018\end{array}$ \\
\hline S1 & & 1 & 1 & 0 & 0 & 0 & 0 & 0 & 1 & 1 & 0 & 0 & 0 & 0 & 0 & \\
\hline 12 & & 1 & 1 & 1 & 1 & 1 & 1 & 1 & 1 & 1 & 0 & 1 & 1 & 1 & 1 & \\
\hline 13 & & 1 & 1 & 1 & 1 & 0.5 & 0.5 & 0.5 & 1 & 0.5 & 0 & 1 & 1 & 0.5 & 0.5 & \\
\hline 14 & & 1 & 1 & 0 & 1 & 1 & 1 & 0 & 1 & 1 & 0.5 & 1 & 1 & 1 & 0 & \\
\hline S2 & & 1 & 1 & 1 & 0 & 0 & 1 & 1 & 1 & 1 & 1 & 1 & 0 & 1 & 0 & \\
\hline 15 & & 1 & 1 & 1 & 1 & 1 & 1 & 1 & 1 & 1 & 0 & 1 & 0 & 1 & 1 & \\
\hline 16 & & 1 & 1 & 0 & 0 & 0 & 0 & 0 & 1 & 1 & 0 & 1 & 1 & 1 & 1 & \\
\hline 17 & & 1 & 1 & 1 & 1 & 1 & 0.5 & 1 & 1 & 1 & 1 & 1 & 1 & 1 & 1 & \\
\hline S3 & & 1 & 1 & 1 & 1 & 1 & 1 & 1 & 1 & 1 & 1 & 1 & 1 & 1 & 1 & \\
\hline S4 & & 0.5 & 0.5 & 0.5 & 0.5 & 0.5 & 0.5 & 0.5 & 0.5 & 0.5 & 0.5 & 0.5 & 0.5 & 0.5 & 0.5 & \\
\hline 18 & & 1 & 1 & 1 & 1 & 1 & 1 & 1 & 1 & 1 & 0.5 & 1 & 1 & 0.5 & 1 & \\
\hline 19 & & 1 & 1 & 1 & 1 & 1 & 1 & 1 & 1 & 1 & 0 & 1 & 1 & 0.5 & 1 & \\
\hline 20 & & 1 & 1 & 1 & 1 & 1 & 1 & 1 & 1 & 1 & 0 & 1 & 1 & 0 & 1 & \\
\hline 21 & & 1 & 1 & 1 & 1 & 1 & 1 & 1 & 1 & 1 & 1 & 1 & 1 & 0.5 & 1 & \\
\hline S5 & & 1 & 1 & 1 & 0 & 1 & 1 & 1 & 1 & 1 & 1 & 1 & 1 & 0 & 0 & \\
\hline 22 & & 0 & 1 & 1 & 1 & 0 & 1 & 1 & 0 & 1 & 0 & 1 & 0 & 0 & 0 & \\
\hline 23 & & 1 & 1 & 0 & 0 & 0 & 0 & 0 & 0 & 1 & 0 & 0 & 0 & 1 & 1 & \\
\hline 24 & & 1 & 1 & 1 & 1 & 1 & 1 & 1 & 1 & 1 & 0 & 1 & 1 & 1 & 1 & \\
\hline 25 & & 1 & 1 & 1 & 1 & 1 & 1 & 1 & 1 & 1 & 0 & 1 & 1 & 1 & 1 & \\
\hline 26 & & 1 & 1 & 1 & 1 & 1 & 1 & 1 & 1 & 1 & 1 & 1 & 1 & 1 & 1 & \\
\hline 27 & & 1 & 1 & 1 & 0 & 0 & 1 & 1 & 1 & 0 & 1 & 1 & 1 & 1 & 1 & \\
\hline Score & & 29 & 27.5 & 24.5 & 21.5 & 20.5 & 25 & 25 & 28.5 & 27 & 13.5 & 28 & 24.5 & 23.5 & 19 & $\begin{array}{l}24.5 / \\
23.62\end{array}$ \\
\hline
\end{tabular}

TABLE 6: Summary of reporting quality assessment.

\begin{tabular}{|c|c|c|c|c|c|c|c|c|}
\hline \multirow[b]{2}{*}{ Section } & \multirow[b]{2}{*}{ Item } & & \multicolumn{2}{|c|}{ Completely reported } & \multicolumn{2}{|c|}{ Partially reported } & \multicolumn{2}{|c|}{ Not reported } \\
\hline & & & $\begin{array}{l}\text { Frequency } \\
(\%)\end{array}$ & $95 \% \mathrm{CI}$ & $\begin{array}{c}\text { Frequency } \\
(\%)\end{array}$ & $95 \% \mathrm{CI}$ & $\begin{array}{c}\text { Frequency } \\
(\%)\end{array}$ & $95 \% \mathrm{CI}$ \\
\hline Title & 1 & Title & $29(100 \%)$ & & 0 & & 0 & \\
\hline Abstract & 2 & Structured summary & $5(17.24 \%)$ & $(-0.16,0.50)$ & $24(82.76 \%)$ & $(0.68,0.98)$ & 0 & \\
\hline Introduction & $\begin{array}{l}3 \\
4\end{array}$ & $\begin{array}{l}\text { Rationale } \\
\text { Objectives }\end{array}$ & $\begin{array}{l}26(89.65 \%) \\
28(96.55 \%)\end{array}$ & $\begin{array}{l}(0.78,1.01) \\
(0.90,1.03)\end{array}$ & $\begin{array}{l}3(10.34 \%) \\
1(3.45 \%)\end{array}$ & $\begin{array}{l}(-0.24,0.45) \\
(-0.32,0.39)\end{array}$ & $\begin{array}{l}0 \\
0\end{array}$ & \\
\hline \multirow{12}{*}{ Methods } & $\begin{array}{l}5 \\
6 \\
7\end{array}$ & $\begin{array}{c}\text { Protocol and registration } \\
\text { Eligibility criteria } \\
\text { Information sources }\end{array}$ & $\begin{array}{c}5(17.24 \%) \\
19(65.52 \%) \\
22(75.86 \%)\end{array}$ & $\begin{array}{c}(-0.16,0.50) \\
(0.44,0.87) \\
(0.58,0.94)\end{array}$ & $\begin{array}{c}0 \\
10(34.48 \%) \\
5(17.24 \%)\end{array}$ & $\begin{array}{l}(0.05,0.64) \\
(-0.16,0.50)\end{array}$ & $\begin{array}{c}24(82.76 \%) \\
0 \\
0\end{array}$ & $(0.68,0.98)$ \\
\hline & 8 & Search & $12(41.38 \%)$ & $(0.14,0.69)$ & 0 & & $17(58.62 \%)$ & $(0.35,0.82)$ \\
\hline & 9 & Study selection & $22(75.86 \%)$ & $(0.58,0.94)$ & $2(6.70 \%)$ & $(-0.28,0.42)$ & $5(17.24 \%)$ & $(-0.16,0.50)$ \\
\hline & 10 & Data collection process & $5(17.24 \%)$ & $(-0.16,0.50)$ & $23(79.31 \%)$ & $(0.63,0.96)$ & $1(3.45 \%)$ & $(-0.32,0.39)$ \\
\hline & 11 & Data items & $3(10.34 \%)$ & $(-0.24,0.45)$ & $26(89.65 \%)$ & $(0.78,1.01)$ & 0 & \\
\hline & $\mathrm{S} 1$ & Geometry of the network & $16(55.17 \%)$ & $(0.31,0.80)$ & 0 & & $13(44.83 \%)$ & $(0.18,0.72)$ \\
\hline & 12 & $\begin{array}{l}\text { Risk of bias within } \\
\text { individual studies }\end{array}$ & $28(96.55 \%)$ & $(0.90,1.03)$ & 0 & & $1(3.45 \%)$ & $(-0.32,0.39)$ \\
\hline & 13 & Summary measures & $18(62.07 \%)$ & $(0.40,0.85)$ & $11(37.93 \%)$ & $(0.09,0.67)$ & 0 & \\
\hline & 14 & $\begin{array}{l}\text { Planned methods of } \\
\text { analysis }\end{array}$ & $23(79.31 \%)$ & $(0.63,0.96)$ & $1(3.45 \%)$ & $(-0.32,0.39)$ & $5(17.24 \%)$ & $(-0.16,0.50)$ \\
\hline & S2 & $\begin{array}{l}\text { Assessment of } \\
\text { inconsistency }\end{array}$ & $23(79.31 \%)$ & $(0.63,0.96)$ & 0 & & $6(20.69 \%)$ & $(-0.12,0.53)$ \\
\hline & 15 & Risk of bias across studies & $22(75.86 \%)$ & $(0.58,0.94)$ & 0 & & $7(24.14 \%)$ & $(-0.08,0.56)$ \\
\hline & 16 & Additional analyses & $14(48.27 \%)$ & $(0.29,0.81)$ & 0 & & $15(51.72 \%)$ & $(0.26,0.77)$ \\
\hline
\end{tabular}


TABLE 6: Continued.

\begin{tabular}{|c|c|c|c|c|c|c|c|c|}
\hline \multirow[b]{2}{*}{ Section } & \multirow[b]{2}{*}{ Item } & & \multicolumn{2}{|c|}{ Completely reported } & \multicolumn{2}{|c|}{ Partially reported } & \multicolumn{2}{|c|}{ Not reported } \\
\hline & & & $\begin{array}{l}\text { Frequency } \\
\quad(\%)\end{array}$ & $95 \% \mathrm{CI}$ & $\begin{array}{l}\text { Frequency } \\
\quad(\%)\end{array}$ & $95 \% \mathrm{CI}$ & $\begin{array}{l}\text { Frequency } \\
\quad(\%)\end{array}$ & $95 \% \mathrm{CI}$ \\
\hline \multirow{10}{*}{ Results } & 17 & Study selection & $25(86.21 \%)$ & $(0.73,0.99)$ & $4(13.79 \%)$ & $(-0.20,0.48)$ & 0 & \\
\hline & S3 & $\begin{array}{c}\text { Presentation of network } \\
\text { structure }\end{array}$ & $18(62.07 \%)$ & $(0.40,0.85)$ & 0 & & $1(3.45 \%)$ & $(-0.32,0.39)$ \\
\hline & S4 & $\begin{array}{l}\text { Summary of network } \\
\text { geometry }\end{array}$ & $1(3.45 \%)$ & $(-0.32,0.39)$ & $26(89.65 \%)$ & $(0.78,1.01)$ & $2(6.70 \%)$ & $(-0.28,0.42)$ \\
\hline & 18 & Study characteristics & $27(93.10 \%)$ & $(0.84,1.03)$ & $2(6.70 \%)$ & $(-0.28,0.42)$ & 0 & \\
\hline & 19 & Risk of bias within studies & $26(89.65 \%)$ & $(0.78,1.01)$ & $1(3.45 \%)$ & $(-0.32,0.39)$ & $2(6.70 \%)$ & $(-0.28,0.42)$ \\
\hline & 20 & Results of individual studies & $17(58.62 \%)$ & $(0.35,0.82)$ & 0 & & $12(41.38 \%)$ & $(0.14,0.69)$ \\
\hline & 21 & Synthesis of results & $27(93.10 \%)$ & $(0.84,1.03)$ & $2(6.70 \%)$ & $(-0.28,0.42)$ & 0 & \\
\hline & S5 & $\begin{array}{l}\text { Exploration for } \\
\text { inconsistency }\end{array}$ & $21(72.41 \%)$ & $(0.53,0.92)$ & 2 (ondor & & $8(27.59 \%)$ & $(-0.03,0.59)$ \\
\hline & 22 & Risk of bias across studies & $13(44.83 \%)$ & $(0.18,0.72)$ & 0 & & $16(55.17 \%)$ & $(0.31,0.80)$ \\
\hline & 23 & $\begin{array}{c}\text { Results of additional } \\
\text { analyses }\end{array}$ & $8(27.59 \%)$ & $(-0.03,0.59)$ & 0 & & $21(72.41 \%)$ & $(0.53,0.92)$ \\
\hline \multirow{4}{*}{ Discussion } & 24 & Summary of evidence & $25(86.21 \%)$ & $(0.73,0.99)$ & $1(3.45 \%)$ & $(-0.32,0.39)$ & $3(10.34 \%)$ & $(-0.24,0.45)$ \\
\hline & 25 & Limitations & $28(96.55 \%)$ & $(0.90,1.03)$ & 0 & & $1(3.45 \%)$ & $(-0.32,0.39)$ \\
\hline & 26 & Conclusions & $27(93.10 \%)$ & $(0.84,1.03)$ & $2(6.70 \%)$ & $(-0.28,0.42)$ & 0 & \\
\hline & 27 & Funding & $21(72.41 \%)$ & $(0.53,0.92)$ & 0 & & $8(27.59 \%)$ & $(-0.03,0.59)$ \\
\hline
\end{tabular}

was no guarantee that all relevant literatures were identified. Finally, since only Chinese and English studies were included, there may be a lack of data to influence the results.

\section{Conclusion}

The NMAs methodological and report quality related to acupuncture and moxibustion were general, and there was still room for improvement in some aspects. For example, the researchers should design the scheme in advance before carrying out the study, design and carry out the study strictly in accordance with PICOS, and present the network structure, so as to improve the prospective and reliability of the study. Considering the importance of PRISMA-NMA checklist to NMA, we advise that the researchers should strictly follow the PRISMA-NMA checklist when writing a NMA.

\section{Abbreviations}

AMSTAR2: A measurement tool to assess the methodological quality of systematic reviews

PRISMA- PRISMA extension statement for reporting of NMA: $\quad$ systematic reviews incorporating network meta-analysis of health care interventions

NMA: Network meta-analysis.

\section{Data Availability}

The data used to support the findings of this study are available from the corresponding author upon request.

\section{Conflicts of Interest}

The authors declare that they have no conflicts of interest.

\section{Authors' Contributions}

JX obtained funds for this study. JX and TY made contributions to the conception and design of the review. FYJ revised the search strategy. TY, JY, and XW searched, extracted, and analyzed data for studies. YT, JY, XW, and JX conducted methodological and reporting quality evaluation. FYJ, HXZ, KL, and LLX assisted. Ting Yuan wrote the original draft. All authors reviewed the manuscript, agreed to all the contents, and agreed the submission.

\section{Acknowledgments}

This study was supported by Jiangxi University of Traditional Chinese Medicine 1050 youth talent project (5141900101), "One Thousand Talents Plan" for Introducing and Training High-level Innovative and Entrepreneurial Talents in Jiangxi Province-The First Batch of Training High-level Scientific and Technological Innovative Talents (Youth) Project (jxsq2019201104), and Natural Science Youth Foundation Key Projects of Jiangxi Province (20192ACB21007).

\section{References}

[1] J. Vas, C. Méndez, E. Perea-Milla et al., “Acupuncture as a complementary therapy to the pharmacological treatment of osteoarthritis of the knee: randomised controlled trial," BMJ, vol. 329, no. 7476, p. 1216, 2004.

[2] G. Lu and A. E. Ades, "Combination of direct and indirect evidence in mixed treatment comparisons," Statistics in Medicine, vol. 23, no. 20, pp. 3105-3124, 2004.

[3] J. P. A. Ioannidis, "Integration of evidence from multiple meta-analyses: a primer on umbrella reviews, treatment networks and multiple treatments meta-analyses," Canadian Medical Association Journal, vol. 181, no. 8, p. 488, 2009.

[4] A. W. Lee, "Review of mixed treatment comparisons in published systematic reviews shows marked increase since 
2009," Journal of Clinical Epidemiology, vol. 67, no. 2, p. 138, 2014.

[5] T. Li, M. A. Puhan, S. S. Vedula et al., "Network meta-analysis highly attractive but more methodological research is needed," BMC Medicine, vol. 9, no. 79, 2011.

[6] J. P. Jansen, B. Crawford, G. W. Bergman et al., "Bayesian meta-analysis of multiple treatment comparisons: an introduction to mixed treatment comparisons: an introduction to mixed tment comparisons," Value in Health, vol. 11, no. 5, pp. 956-964, 2008.

[7] A. Stam, J. P. T. Higgins, J. R. Geddes, and G. Salanti, "Conceptual and technical challenges in network metaanalysis," Annals of Internal Medicine, vol. 159, no. 2, p. 130, 2013.

[8] G. Salanti, J. P. Higgins, A. Ades, and J. P. Ioannidis, "Evaluation of networks of randomized trials," Statistical Methods in Medical Research, vol. 17, no. 3, pp. 279-301, 2008.

[9] L. Li, J. Tian, H. Tian et al., "Network meta-analyses could be improved by searching more sources and by involving a librarian," Journal of Clinical Epidemiology, vol. 67, no. 9, p. 1001, 2014.

[10] G. Salanti, "Indirect and mixed-treatment comparison, network, or multiple-treatments meta-analysis: many names, many benefits, many concerns for the next generation evidence synthesis tool," Research Synthesis Methods, vol. 3, no. 2, pp. 80-97, 2012.

[11] R. C. Lorenz, K. Matthias, D. Pieper et al., "A psychometric study found AMSTAR 2 to be a valid and moderately reliable appraisal tool," Journal of Clinical Epidemiology, vol. 114, p. 133, 2019.

[12] B. Hutton, G. Salanti, D. M. Caldwell et al., "The PRISMA extension statement for reporting of systematic reviews incorporating network meta-analyses of health care interventions: checklist and explanations," Annals of Internal Medicine, vol. 162, no. 11, p. 777, 2015.

[13] J. Tian, J. Zhang, L. Ge, K. Yang, and F. Song, "The methodological and reporting quality of systematic reviews from China and the USA are similar," Journal of Clinical Epidemiology, vol. 85, p. 50, 2017.

[14] J. L. Li, L. Ge, J. C. Ma et al., "Quality of reporting of systematic reviews published in "evidence-based" Chinese journals," Systematic Reviews, vol. 3, no. 58, 2014.

[15] Y. Fu, L. Dong, J. Pan et al., "A meta-analysis of TCM external therapy and conventional western medicine oral therapy for acute gout arthritis," Chinese Journal of Clinical Medicine, vol. 31, no. 1, pp. 94-101, 2019.

[16] M. Ding, "A meta-analysis of the effectiveness of external Chinese medicine in relieving postoperative abdominal distension," Journal of Guangzhou University of Chinese Medicine, vol. 36, no. 7, pp. 1039-1044, 2019.

[17] S. Li, "A meta-analysis of Chinese medicine combined therapy for post-stroke depression," Modern Distance Education of Chinese Medicine, vol. 16, no. 3, pp. 148-150, 2018.

[18] Z. Bu, X. Luo, and C. Tang, "Systematic evaluation of the effectiveness of acupuncture and moxibustion in treating optic nerve atrophy and meta-analysis of the mesh," Journal of Beijing University of Chinese Medicine, vol. 40, no. 5, pp. $428-435,2017$.

[19] X. Zhang, "Efficacy of acupuncture and sulfasalazine in treatment of ankylosing spondylitis: a meta-analysis," Chinese Medical Journal, vol. 36, no. 10, pp. 2321-2324, 2018.

[20] Y. Song, "A meta-analysis of the effects of acupuncture and moxibustion on ovulation rate and pregnancy rate in patients with polycystic ovary syndrome," Chinese Acupuncture and Moxibustion, vol. 39, no. 7, pp. 792-798, 2019.

[21] M. Yang, Q. Shi, and G. Xu, "A meta-analysis of acupuncture and moxibustion treatment of lumbar disc herniation with cupping and massage," Chinese Journal of Traditional Chinese Medicine, vol. 34, no. 9, pp. 2153-2157, 2016.

[22] G. Li, "A meta-analysis of acupuncture therapy for primary dysmenorrhea," Chinese Journal of Evidence-Based Medicine, vol. 17, no. 10, pp. 1212-1223, 2017.

[23] W Jia, C. Wang, and Y. Yin, "A meta-analysis of acupoint catgut embedding for chronic urticaria," Chinese Journal of External Medicine, vol. 27, no. 5, pp. 44-49, 2018.

[24] Y. Liu, "Efficacy of small needle knife and hyaluronic acid in the treatment of knee osteoarthritis: a meta-analysis of mesh," Journal of Hubei University of Medicine, vol. 35, no. 2, pp. 157-164, 2016.

[25] Z. Feng, Mesh Meta-Analysis of Clinical Efficacy of Acupuncture Alone in Treating Primary Dysmenorrhea, Inner Mongolia Medical University, Hohhot, China, 2018.

[26] F. Liu, "A meta-analysis of different acupuncture treatments for shoulder pain after stroke," Chinese Archives of Traditional Chinese Medicine, vol. 37, no. 3, pp. 533-538, 2019.

[27] H.-S. Yang, Y.-G. Fang, H.-F. Xu, X.-T. Li, J. Shang, and Y.-Q. Yin, "Systematic evaluation on the clinical efficacy of acupoint stimulation therapy for treatment of premature ovarian insufficiency on the basis of network Meta-analysis," World Journal of Acupuncture-Moxibustion, vol. 27, no. 3, pp. 26-39, 2017.

[28] L. Zhu, Y. Ma, and X. Deng, "Comparison of acupuncture and other drugs for chronic constipation: a network meta-analysis," PLoS One, vol. 13, no. 4, Article ID 196128, 2018.

[29] H. Zheng, "Nonpharmacological conservative treatments for chronic functional constipation: a systematic review and network meta-analysis," Neurogastroenterol Motil, vol. 31, no. 1, Article ID 13441, 2019.

[30] Z. Qin, "Network meta-analysis of the efficacy of acupuncture, alpha-blockers and antibiotics on chronic prostatitis/chronic pelvic pain syndrome," Scientific Reports, vol. 6, p. 35737, 2016.

[31] S. Li, P. Xie, Z. Liang et al., "Efficacy comparison of five different acupuncture methods on pain, stiffness, and function in osteoarthritis of the knee: a network meta-analysis," Evidence-Based Complementary and Alternative Medicine, vol. 2018, Article ID 1638904, 19 pages, 2018.

[32] Z. Mo, D. Li, R. Zhang et al., "Comparisons of the effectiveness and safety of tuina, acupuncture, traction, and Chinese herbs for lumbar disc herniation: a systematic review and network meta-analysis," Evidence-Based Complementary and Alternative Medicine, vol. 2019, Article ID 6821310, 10 pages, 2019.

[33] F. Luo, X. Huang, X. Liu, L. Wang, and N. Xu, "Comparative efficacy and safety of NSAIDs-controlled acupuncture in the treatment of patients with primary dysmenorrhoea: a Bayesian network meta-analysis," Journal of International Medical Research, vol. 47, no. 1, pp. 19-30, 2019.

[34] M.-L. Yeh, S.-H. Ko, M.-H. Wang, C.-C. Chi, and Y.-C. Chung, "Acupuncture-related techniques for psoriasis: a systematic review with pairwise and network meta-analyses of randomized controlled trials," The Journal of Alternative and Complementary Medicine, vol. 23, no. 12, pp. 930-940, 2017.

[35] Y. Y. Chen, J. Li, M. Chen, L. Yue, T.-W. She, and H. Zheng, "Acupuncture versus propranolol in migraine prophylaxis: an indirect treatment comparison meta-analysis," Journal of Neurology, vol. 267, no. 1, pp. 14-25, 2019. 
[36] X. Tan, Y. Pan, W. Su et al., "Acupuncture therapy for essential hypertension: a network meta-analysis," Annals of Translational Medicine, vol. 7, no. 12, p. 266, 2019.

[37] X. Li, R. Wang, and X. Xing, "Acupuncture for myofascial pain syndrome: a network meta-analysis of 33 randomized controlled trials," Pain Physician, vol. 20, no. 6, pp. 883-902, 2017.

[38] L. Zhu, Y. Ma, S. Ye, and Z. Shu, "Acupuncture for diarrhoeapredominant irritable bowel syndrome: a network metaanalysis," Evidence-Based Complementary and Alternative Medicine, vol. 2018, Article ID 2890465, 12 pages, 2018.

[39] W. J. Xiong and W. Chen, "Acupuncture for diabetic peripheral neuropathy: a network meta-analysis," Journal of Alternative and Complementary Medicine, vol. 22, no. 6, pp. A123-A124, 2016.

[40] F.-W. Yang, R. Feng, H.-C. Wang et al., "Acupuncture and related therapies used as add-on to conventional treatments for heart failure: a systematic review of pairwise and network meta-analyses," World Journal of Acupuncture-Moxibustion, vol. 28, no. 4, pp. 268-277, 2018.

[41] Y. Zhang, J. Li, G. Mo et al., "Acupuncture and related therapies for obesity: a network meta-analysis," EvidenceBased Complementary and Alternative Medicine, vol. 2018, Article ID 9569685, 20 pages, 2018.

[42] M. S. Corbett, S. J. C. Rice, V. Madurasinghe et al., "Acupuncture and other physical treatments for the relief of pain due to osteoarthritis of the knee: network meta-analysis," Osteoarthritis and Cartilage, vol. 21, no. 9, pp. 1290-1298, 2013.

[43] T. Wang, C. Xu, K. Pan, and H. Xiong, "Acupuncture and moxibustion for chronic fatigue syndrome in traditional Chinese medicine: a systematic review and meta-analysis," BMC Complementary and Alternative Medicine, vol. 17, no. 1, p. 163, 2017.

[44] F. Yang, H. Wang, J. Zou et al., “Assessing the methodological and reporting quality of network meta-analyses in Chinese medicine," Medicine, vol. 97, no. 47, p. 13052, 2018.

[45] F. Yang, J. Zhang, and B. Zhang, "Meta analysis quality evaluation of Chinese medicine network," Chinese Journal of Chinese Medicine, vol. 33, no. 10, pp. 4599-4606, 2018. 